\title{
Variability in fMRI: An Examination of Intersession Differences
}

\author{
D. J . McGonigle, A. M. Howseman, B. S. Athwal, K. J . Friston, R. S. J . Frackowiak, and A. P. Holmes ${ }^{1}$ \\ Well come Department of Cognitive Neurology, Institute of Neurology, London WC1N 3BG, United Kingdom
}

Received September 22, 1999

The results from a single functional magnetic resonance imaging session are typically reported as indicative of the subject's functional neuroanatomy. Underlying this interpretation is the implicit assumption that there are no responses specific to that particular session, i.e., that the potential variability of response between sessions is negligible. The present study sought to examine this assumption empirically. A total of 99 sessions, comprising 33 repeats of simple motor, visual, and cognitive paradigms, were collected over a period of $\mathbf{2}$ months on a single male subject. For each paradigm, the inclusion of session-by-condition interactions explained a significant amount of error variance ( $P<0.05$ corrected for multiple comparisons) over a model assuming a common activation magnitude across all sessions. However, many of those voxels displaying significant session-by-condition interactions were not seen in a multisession fixed-effects analysis of the same data set; i.e., they were not activated on average across all sessions. Most voxels that were both significantly variable and activated on average across all sessions did not survive a randomeffects analysis (modeling between-session variance). We interpret our results as demonstrating that correct inference about subject responses to activation tasks can be derived through the use of a statistical model which accounts for both within- and between-session variance, combined with an appropriately large session sample size. If researchers have access to only a single session from a single subject, erroneous conclusions are a possibility, in that responses specific to this single session may be claimed to be typical responses for this subject.

\section{INTRODUCTION}

This study assesses the generality of results obtained from a single functional magnetic resonance imaging (fMRI) session. $\mathrm{fMRI}$ is a noninvasive technique that has revolutionized the study of human brain function (e.g., Belliveau et al., 1991; Ogawa et al., 1992;

\footnotetext{
${ }^{1}$ Current address: Robertson Centre for Biostatistics, Boyd Orr Building, University Avenue, Glasgow G12 8QQ, Scotland, UK.
}

Kwong et al., 1992). As with many brain imaging techniques, such as positron emission tomography, a number of observations (scans) from each subject are collected. A single experimental examination of one subject in this fashion constitutes a session. Although exceptions exist, it is unusual for a subject to be scanned on more than one occasion and, more often than not, a single fMRI session is assumed to give an accurate representation of a subject's functional neuroanatomy.

There may be problems, however, if one adopts this "one subject, one session" approach to neuroimaging experiments. One session is only a single, discrete "snapshot" of the subject's brain and may not epitomize responses to the sensorimotor or cognitive challenge employed. I ndeed, differences between sessions are inevitable: for example, the BOLD response is an indirect and semiqualitative measure of neuronal activity, and the relationship between BOLD contrast and cerebral oxygen metabolism is influenced by a number of physiological factors (e.g., for review see Ogawa et al., 1998). Furthermore, single-session results may be influenced by slight variations in the hardware characteristics of the MR scanner, which are not systematic across sessions (e.g., the shim performed to homogenize the $B_{0}$ field of the scanner; Howseman et al., 1998). Any differences in subject position within the headcoil on separate scanning sessions may also result in greater variability in voxel signal changes, due to partial volume effects, as may different patterns of subject movement between sessions. In addition to the above, nonspecific physiological effects such as the level of arousal may further influence the neurovascular re sponse to the activation task in question.

These effects are hard to control and may substantially influence a single session's results, such that the experiment may ultimately say as much about the context under which the data were acquired as the effects of the experimental manipulation itself. Although few researchers would expect a precise replication of the results if an experiment were repeated, it is currently unclear how generalizable single-session results are with fMRI. 


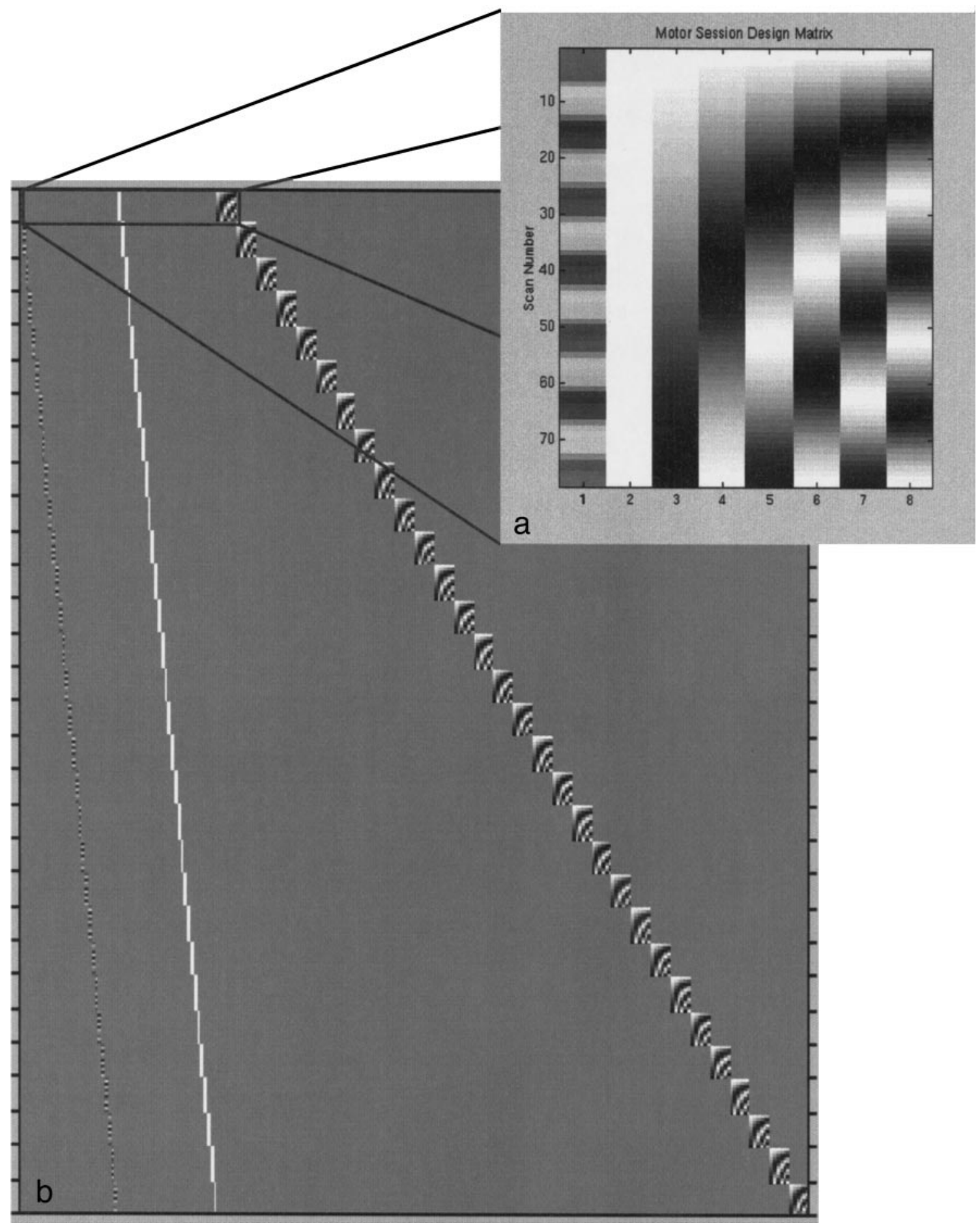

FIG. 1. Design matrices used for analysis. The design matrix is a graphical representation of the experimental model. Each column of the design matrix represents a separate regressor within the statistical model, and each row represents a singlefMRI volume. The gray-scale color value within each cell displays the value of the relevant regressor at that point in the fM RI time series before the model fit is estimated. For example, a simple boxcar regressor, before convolution, would occupy a single design matrix column, and each cell within the column would have a value of 0 or 1 , depending on the experimental design used. (A) A single session design matrix with the regressor of interest (the $\mathrm{CBC}$, column 1), the session mean effect ( $\gamma_{i}$, column 2), and the set of discrete cosine basis functions used to effect high-pass filtering (columns 3-8). (B) A multisession design matrix, constructed from $n$ single-session design matrices, where $n$ is the number of sessions analyzed at the multisession level. The design matrix in A was used only for single-session analyses, whereas B was used for both the fixedand the random-effects multisession analyses. 

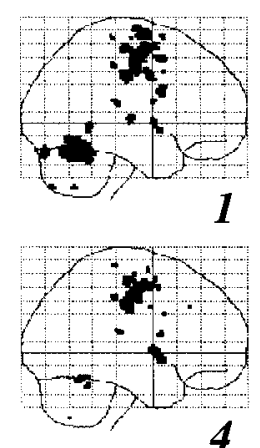

4

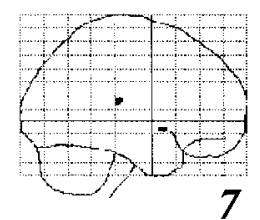

7

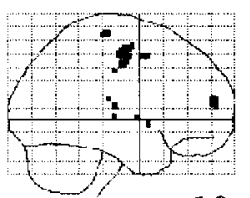

10

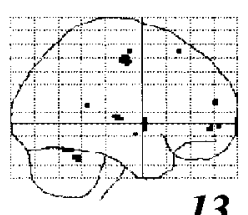

13
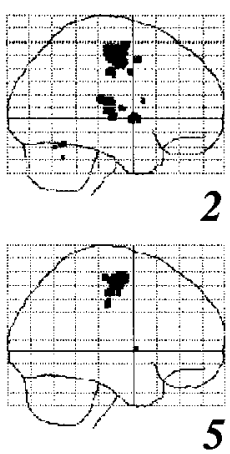

5
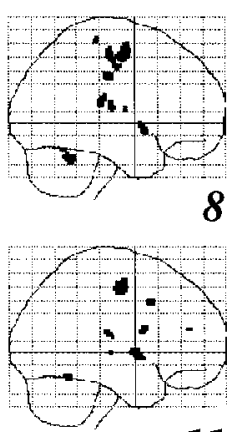

11

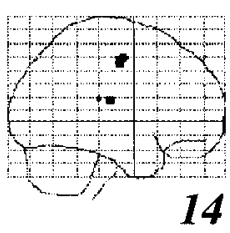

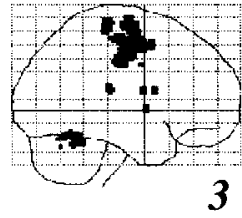

3
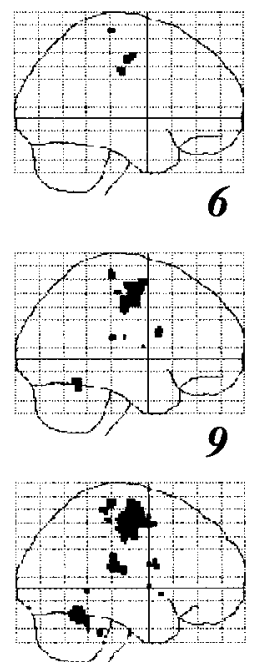

12

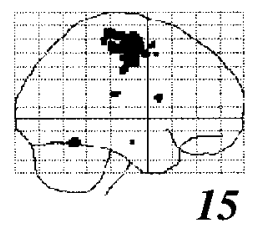

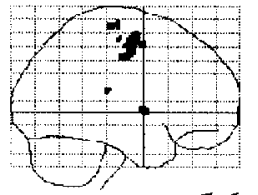

16

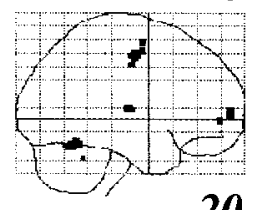

20

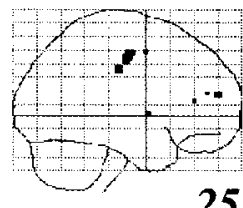

25

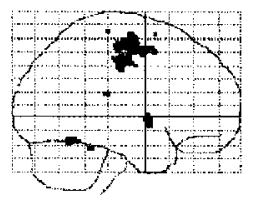

28

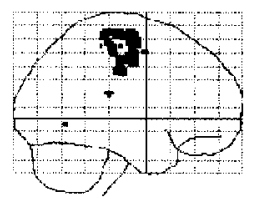

31

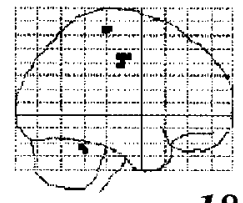

18

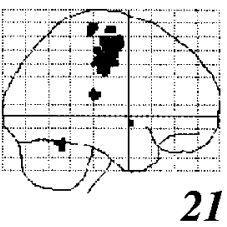

21

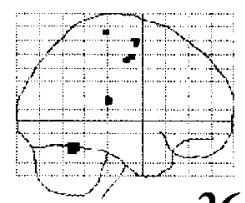

26

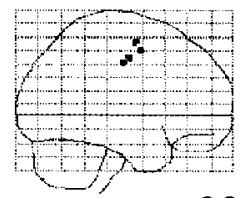

29

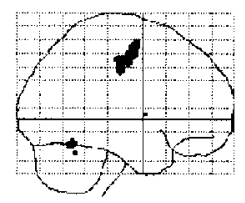

32

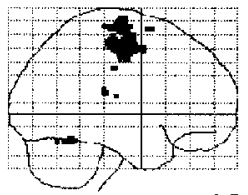

19

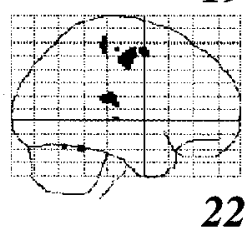

22

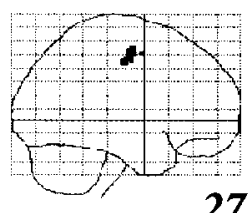

27

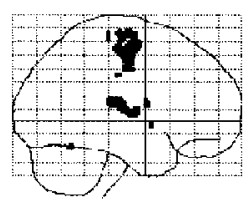

30

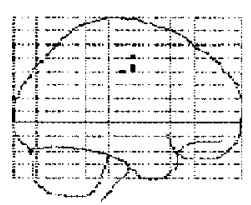

33

FIG. 2. Single-session sagittal MIPs for the motor paradigm. The number of each session is displayed bel ow it. Although 33 sessions were collected, only 30 are shown here (sessions 17,23 , and 24 were rejected due to movement artifacts). All results are thresholded at $P<0.05$ corrected for multiple comparisons unless otherwise stated.

This influence of session context on the activation effects of a study constitutes a session-by-condition interaction. Although a number of studies have examined the reproducibility of $\mathrm{fMRI}$ across a small number of sessions (Cohen et al., 1999; Noll et al., 1997; Rombouts et al., 1998; Tegeler et al., 1999; Yetkin et al., 1996), our primary aim was to examine how well a single session typifies a subject's responses. J ust as the significance of within-session experimental effects is assessed by sampling a number of scans for each condition, to assess between-session differences one must sample multiple sessions. If a single session is to be a good exemplar of a subject's functional neuroanatomy, session-by-condition interactions must be minimal.

The issue of single-session generality also influences data analysis. If activation effects do indeed vary substantially between sessions, to generalize the results to the subject an experiment will need to utilize multiple sessions and assess the data accounting for both within- and between-session variability. Typically, these two levels of variability are not addressed, even if multiple sessions are acquired; the experimental ef- fects of interest are assessed using statistical models that utilize within-session error variance (residual scan/scan variability) as the only component of variance. Although session-by-condition interactions are often modeled, the variability of these interaction effects does not enter into the inference. Such a model, employing a single variance component, is a fixed-effects model (Searle et al., 1992). These models have been the norm in neuroimaging analysis and assess only the average experimental effect across the observed sessions. They do not take account of the variability of responses between sessions and therefore cannot be used to draw conclusions about a subject's typical response. For example, a spuriously large activation in one voxel during only one session may be large enough to dominate that voxel's average re sponses across sessions. In the case of a single session collected from a single subject, the experiment is reduced to a case study. Conclusions regarding the subject's typical response can be made only under the implicit assumption that intersession variability of re sponse would be negligible were the experimental ses- 

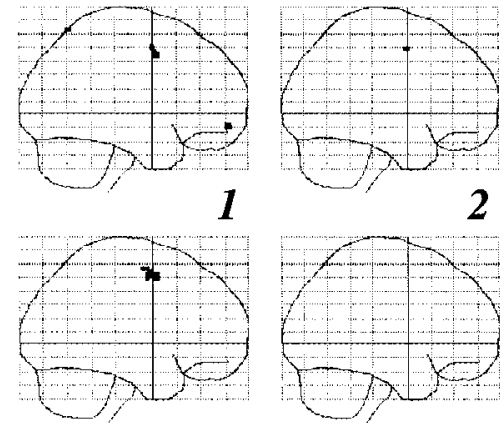

4
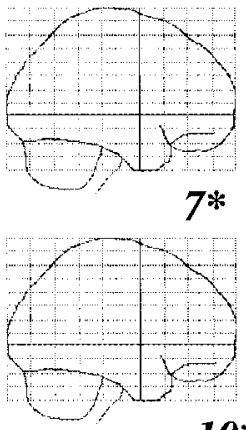

$10 *$

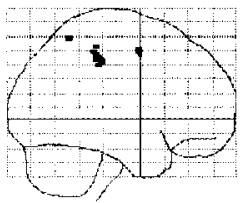

13
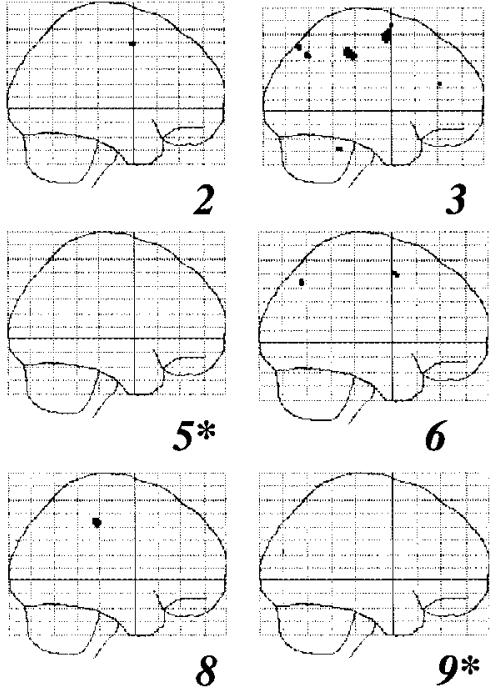

3

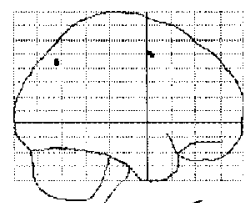

6

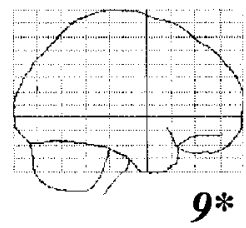

9*

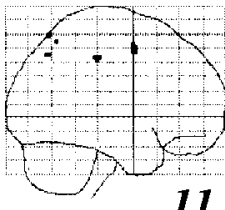

11

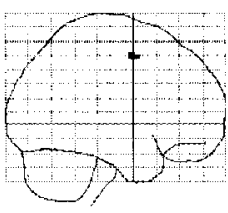

14

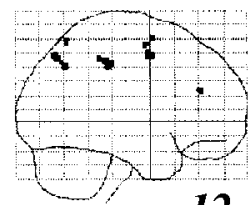

12

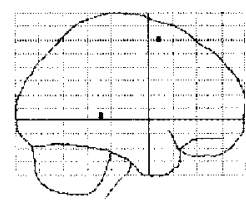

15

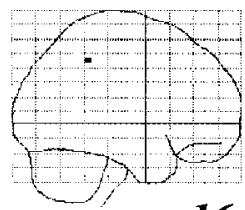

16

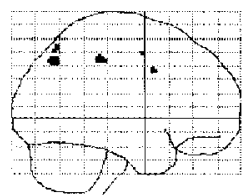

19

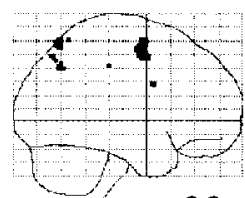

22

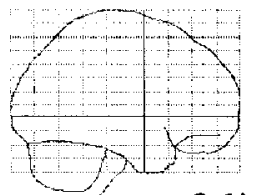

$26^{*}$

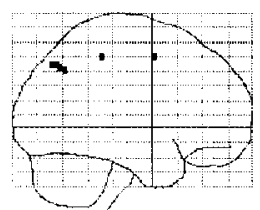

31

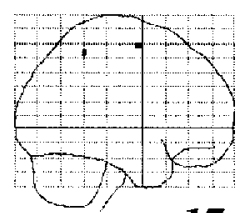

17

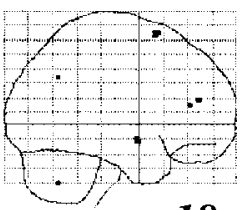

18

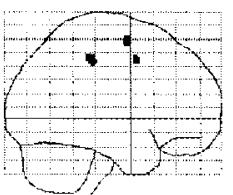

20

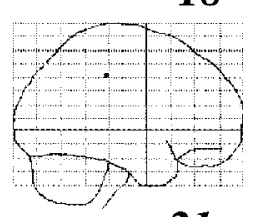

21

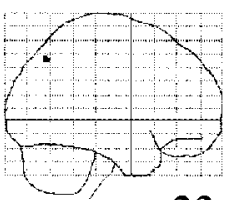

23

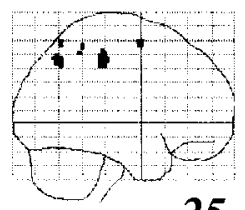

25

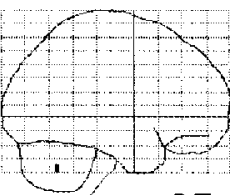

27

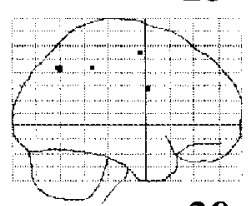

29

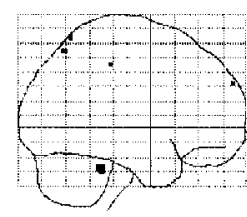

32

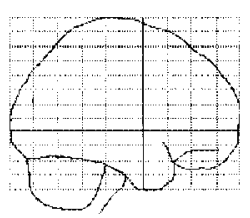

$33 *$

FIG. 3. Single-session sagittal MIPs for the cognitive paradigm. Similar to Fig. 2, although 33 sessions were collected, only 30 are displayed. Sessions marked with "*" contain no significant voxels.

sion repeated. As discussed above, this is highly unlikely.

If session-by-condition interactions are substantial, random-effects models are required. Random-effects models allow for multiple variance components (Searle et al., 1992), so the effects of each session on the BOLD response are treated as a random variable. This reflects the fact that a single session is considered a sample from the population of all possible sessions from the subject, and so significance can be computed, accounting for both between- and within-session variance. Random-effects analyses have previously been employed to account for between-subject variability, or subject-by-condition interactions, in fMRI studies (Holmes et al., 1998; Henson et al., 1999a,b).

As the random-effect analysis infers about the population from which the samples were drawn, the $\mathrm{N}$ of observations is now the number of sessions. As the number of sessions is quite small, these analyses tend to have low power, that is, there is a high chance of type II errors. An analysis of this type, however, is essential for the correct level of inference if session-bycondition interactions are considerable. In the present study we examined the reproducibility of the BOLD response in a single subject over multiple sessions for simple motor, cognitive, and visual paradigms. We first present results from each session analyzed in isolation, as if from a single-session experiment, using only within-session variance to compute significance. We then show where significant session-by-condition interactions occur for each of our activation paradigms. Finally, we consider two analyses of the entire multisession data set. The first is a fixed-effects analysis, the second a simple random-effects analysis.

\section{METHODS}

\section{Subject and Session Details}

The subject was a healthy 23-year-old right-handed male. As our goal in the current study was to examine the generality of a single session, each session was conducted as if it were the first time the subject had been examined: in effect, as if only one session was to be obtained. Our motivation was therefore to control for obvious and artifactual between-session differences 


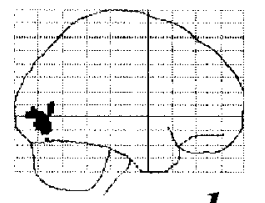

I

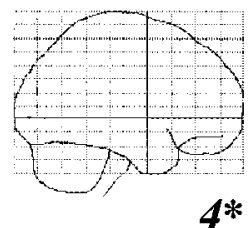

$4^{*}$

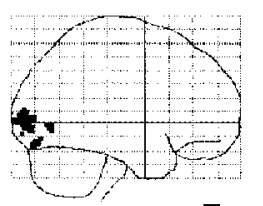

7

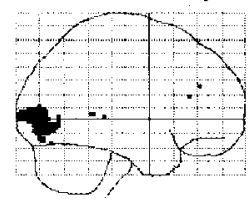

10

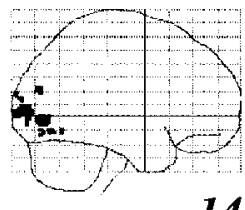

14

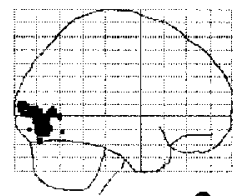

2

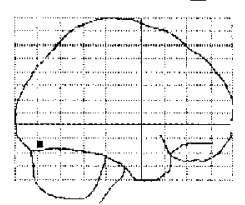

5

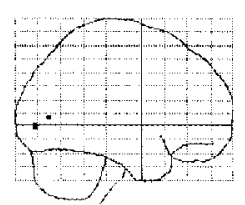

8

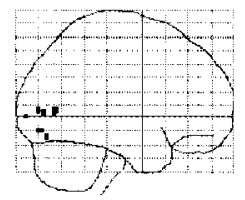

11

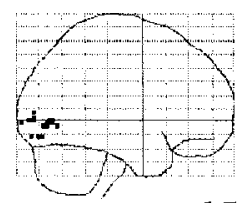

15

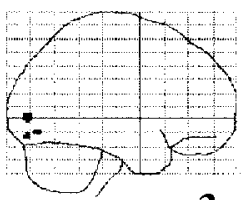

3

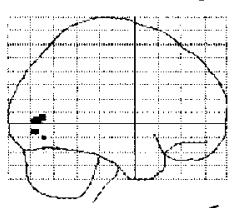

6

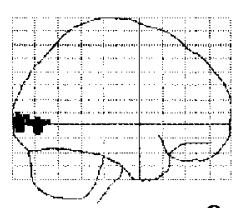

9

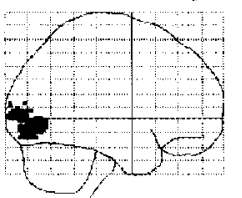

12

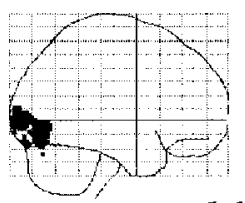

16
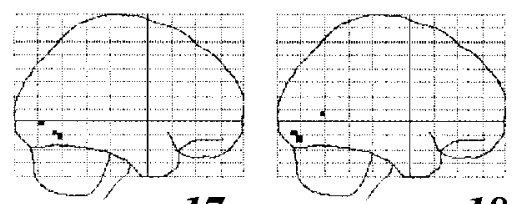

18

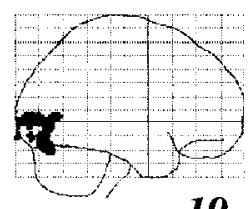

17
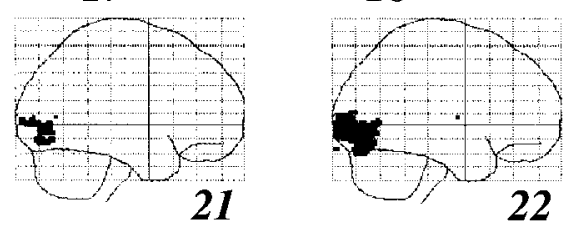

19
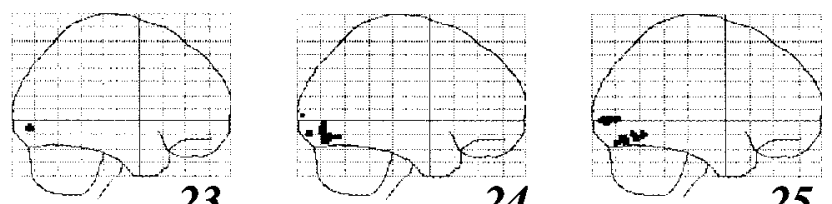

24
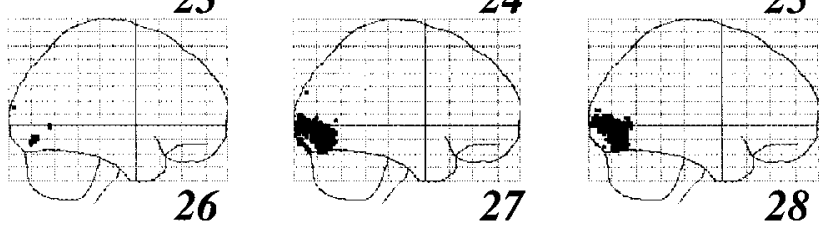

28

FIG. 4. Single-session sagittal MIPs for the visual paradigm. Similar to Figs. 2 and 3 , only 31 sessions are displayed. Sessions marked with "*" contain no significant voxels.

while ensuring that sources of typical between-session variability (scanner hardware and subject physiology) would be sampled in an unbiased manner. The following precautions were taken: the same operators al ways controlled the scanner, ambient light and sound levels were similar between sessions, and spoken instructions to the subject were al ways exactly the same. One obvious factor that we could not control was that our subject was always aware that he had performed the task before in the scanner, only under slightly different circumstances. We called this the "Groundhog Day" effect.

Ninety-nine individual sessions were acquired from the subject over a period of 2 months. Each scanning session consisted of one run of a motor, cognitive, or visual paradigm. To minimize scanning time, sessions were acquired in blocks of three. Each block of three sessions comprised a motor, visual, and cognitive session. The order of sessions across scanning blocks was randomized to balance any possible order effects. Ses- sion paradigms were designed to reduce the effects of variable task performance. For example, the subject was familiarized with both the random number generation and the finger-tapping task before performing them in the scanner, in an attempt to eliminate performance effects. In addition, the rates at which both tasks were performed were chosen to ensure that subject performance would be stable across sessions. These decisions were informed by studies which used similar paradigms (motor paradigm-Blinkenberg et al., 1996; cognitive paradigm—J ahanshahi et al., submitted for publication).

\section{Motor Paradigm}

The subject tapped his right index finger, paced by an auditory tone $(1.5 \mathrm{~Hz})$. The subject's hand was restrained within a custom-built thermoplastic splint, which ensured that the amplitude of the finger movement was consistent both across and within sessions. 
TABLE 1

Talairach coordinates

Area

Cluster size $(\mathrm{k})^{\mathrm{a}}$

Z score ${ }^{b}$

Y

Z

Left precentral gyrus (SM1)

Left precentral gyrus (SM 1)

Left postcentral gyrus (SM1)

Right cerebellum, anterior lobe

Right cerebellum, anterior lobe

Right cerebellum, anterior lobe

Left supplementary motor area (SMA)

Right SMA

Right SMA

Left inferior frontal gyrus

Left inferior frontal gyrus

Right postcentral gyrus (SM1)

Right precentral gyrus (SM1)

Right precentral gyrus (SM1)

Left inferior thalamus

Left ventral midbrain

Left midbrain

Right lateral premotor cortex

Right inferior frontal gyrus

Right inferior parietal lobule

Right transverse temporal gyrus

Right superior temporal gyrus

Left posterior cingulate gyrus

Left posterior cingulate gyrus

Right inferior frontal operculum Right insula

Left cerebellum, anterior lobe

Right cerebellum, anterior lobe Right cerebellar tonsil

Left posterior postcentral gyrus

Left superior frontal gyrus

Left superior thalamus

Left thalamus

Left medial frontal gyrus

Left medial frontal gyrus

Left inferior frontal gyrus

Third ventricle

Right putamen

Right putamen

Right anterior cingulate gyrus

Right postcentral gyrus

Right thalamus

Right inferior frontal gyrus

White matter, subcortical

White matter, subcortical

Right posterior cingulate

Right cerebellum

Right thalamus

Left cerebellum

Left superior frontal gyrus

Right thalamus

Right cerebellum

Left superior frontal gyrus

Right caudate nucleus

(a) Local maxima for the motor fixed-effects model

\begin{tabular}{|c|c|c|c|c|}
\hline \multirow[t]{3}{*}{5958} & 9.77 & -38 & -10 & 52 \\
\hline & 9.63 & -62 & -20 & 38 \\
\hline & 9.51 & -60 & -18 & 46 \\
\hline \multirow[t]{3}{*}{1709} & 9.50 & 20 & -54 & -18 \\
\hline & 9.19 & 2 & -54 & -24 \\
\hline & 9.14 & 8 & -58 & -20 \\
\hline \multirow[t]{3}{*}{927} & 9.39 & -2 & -2 & 52 \\
\hline & 8.75 & 8 & 2 & 58 \\
\hline & 8.50 & 4 & 8 & 66 \\
\hline \multirow[t]{2}{*}{227} & 9.23 & 62 & 6 & 16 \\
\hline & 6.98 & 62 & 4 & 4 \\
\hline \multirow[t]{3}{*}{197} & 9.07 & 58 & -12 & 50 \\
\hline & 8.29 & 52 & -6 & 38 \\
\hline & 7.71 & 62 & -2 & 38 \\
\hline \multirow[t]{3}{*}{466} & 8.84 & -12 & -18 & 2 \\
\hline & 6.15 & -8 & -12 & -14 \\
\hline & 5.90 & -8 & -20 & -12 \\
\hline 70 & 8.80 & 38 & -8 & 50 \\
\hline 62 & 8.60 & 64 & 0 & 16 \\
\hline \multirow[t]{3}{*}{604} & 8.54 & 50 & -28 & 24 \\
\hline & 8.36 & 52 & -18 & 12 \\
\hline & 7.99 & 68 & -26 & 16 \\
\hline \multirow[t]{2}{*}{100} & 8.32 & -8 & -26 & 42 \\
\hline & 7.11 & -2 & -22 & 48 \\
\hline \multirow[t]{2}{*}{228} & 8.23 & 0 & 6 & 4 \\
\hline & 6.12 & 0 & 6 & 10 \\
\hline 66 & 7.85 & 20 & -56 & -20 \\
\hline \multirow[t]{2}{*}{98} & 7.73 & 2 & -58 & -48 \\
\hline & 7.43 & 6 & -70 & -40 \\
\hline 67 & 7.63 & 18 & -46 & 64 \\
\hline 79 & 7.49 & 28 & 42 & 26 \\
\hline \multirow[t]{2}{*}{46} & 7.40 & 18 & -10 & 18 \\
\hline & 5.73 & -12 & -6 & 12 \\
\hline \multirow[t]{3}{*}{223} & 7.27 & -38 & 58 & -2 \\
\hline & 7.27 & -30 & 62 & 4 \\
\hline & 7.20 & -34 & 54 & -8 \\
\hline 22 & 7.27 & 6 & -42 & -6 \\
\hline \multirow[t]{2}{*}{171} & 7.26 & 8 & 6 & -2 \\
\hline & 6.68 & 0 & 6 & -12 \\
\hline 27 & 6.74 & 14 & 8 & 42 \\
\hline 7 & 6.74 & 40 & -30 & 58 \\
\hline 40 & 6.70 & 14 & -14 & 2 \\
\hline 18 & 6.46 & 30 & 60 & -2 \\
\hline \multirow[t]{2}{*}{33} & 6.46 & 16 & 0 & 12 \\
\hline & 5.13 & 12 & 6 & 2 \\
\hline 19 & 6.43 & 12 & -24 & 46 \\
\hline 18 & 5.94 & 48 & -50 & -38 \\
\hline 16 & 5.79 & 20 & 0 & -6 \\
\hline 12 & 5.76 & -36 & -64 & -26 \\
\hline 5 & 5.64 & -28 & 28 & 56 \\
\hline 9 & 5.55 & 8 & -4 & -4 \\
\hline 12 & 5.51 & 40 & -62 & -50 \\
\hline 8 & 5.42 & -22 & 38 & 48 \\
\hline 9 & 5.30 & 20 & -6 & 20 \\
\hline
\end{tabular}

(b) Local maxima for the motor random-effects analysis

Left precentral gyrus (SM1)

Left precentral gyrus (SM1)

Left frontal operculum

Right cerebellum, anterior lobe

Right cerebellum, anterior lobe

Right cerebellum, anterior lobe
4253

951

$\begin{array}{lr}8.76 & -36 \\ 8.45 & -60 \\ 8.37 & -48 \\ 8.04 & 18 \\ 7.55 & 30 \\ 7.34 & 36\end{array}$

$-10$

$-18$

4

$-52$

$-52$

$-62$ 
TABLE 1-Continued

Talairach coordinates

\begin{tabular}{|c|c|c|c|c|c|}
\hline \multirow[b]{2}{*}{ Area } & \multirow[b]{2}{*}{ Cluster size $(k)^{a}$} & \multirow[b]{2}{*}{ Z score ${ }^{b}$} & \\
\hline & & & $x$ & Y & Z \\
\hline Left inferior frontal gyrus & 151 & 8.00 & -62 & 8 & 16 \\
\hline Left inferior precentral gyrus & & 6.96 & -60 & -2 & 16 \\
\hline Left supplementary motor area (SMA) & 545 & 7.62 & -8 & -2 & 48 \\
\hline SMA & & 7.34 & 0 & 2 & 56 \\
\hline Right superior SMA & & 7.15 & 4 & 10 & 66 \\
\hline Left thalamus & 173 & 7.44 & -12 & -20 & 2 \\
\hline Left midbrain (red nucleus) & & 5.47 & -12 & -14 & -10 \\
\hline Right postcentral gyrus (SM1) & 104 & 7.40 & 58 & -12 & 50 \\
\hline Right inferior precentral gyrus & & 6.93 & 50 & -4 & 36 \\
\hline Right inferior precentral gyrus & & 5.75 & 60 & -2 & 40 \\
\hline Left supramarginal gyrus & 44 & 7.27 & -58 & -44 & 18 \\
\hline Right lateral premotor cortex & 46 & 7.19 & 38 & -8 & 52 \\
\hline Right frontal operculum & 104 & 7.10 & 50 & 6 & 4 \\
\hline Right parietal operculum & 256 & 7.07 & 50 & -28 & 26 \\
\hline Right transverse temporal gyrus & & 6.94 & 48 & -18 & 14 \\
\hline Right transverse temporal gyrus & & 6.16 & 68 & -26 & 16 \\
\hline Left posterior cingulate gyrus & 39 & 6.88 & -8 & -24 & 44 \\
\hline Right inferior frontal gyrus & 32 & 6.71 & 64 & 0 & 16 \\
\hline Right inferior postcentral gyrus & 25 & 6.56 & 62 & -12 & 12 \\
\hline Cerebellar vermis & 25 & 6.22 & 6 & -70 & -40 \\
\hline Left superior frontal gyrus & 21 & 6.04 & -28 & 42 & 26 \\
\hline Right cerebellar tonsil & 8 & 5.88 & 12 & -56 & -48 \\
\hline Left superior thalamus & 12 & 5.81 & -20 & -12 & 18 \\
\hline Left posterior postcentral gyrus & 14 & 5.73 & -18 & -46 & 64 \\
\hline Left cerebellum, anterior lobe & 10 & 5.68 & -20 & -56 & -20 \\
\hline Right putamen & 20 & 5.57 & 28 & 8 & -2 \\
\hline
\end{tabular}

Note SM 1, primary somatomotor cortex; SMA, supplementary motor cortex.

${ }^{a}$ Only clusters with $k \geq 5$ are listed. Where multiple foci exist for a cluster, the three most significant are reported.

${ }^{\mathrm{b}}$ All foci reported survive a statistical threshold of $\mathrm{P}<0.05$ corrected for multiple comparisons.

Each activation epoch was alternated with a rest epoch, in which the pacing tone was delivered to control for auditory activation. Thirteen blocks were collected per session ( 7 rest and 6 active). The subject maintained fixation on a cross that was backprojected onto a transparent screen by an LCD video projector. The projector was similarly employed to deliver visual instructions to the subject before each block (either "Move" or "Rest").

\section{Cognitive Paradigm}

The subject generated random numbers from 1 to 9 , paced by an auditory tone $(0.66 \mathrm{~Hz})$. In the rest condition the subject counted from 1 to 9 , similarly paced by the auditory tone. The subject fixated in a fashion similar to that used before. Thirteen epochs were collected in total ( 7 rest and 6 active).

\section{Visual Paradigm}

A reversing black and white checkerboard flickering at $8 \mathrm{~Hz}$ (Fox and Raichle, 1985) was presented to the subject. The subject focused on a central fixation spot that was constant across both activation (reversing checkerboard stimulation) and rest (fixation spot only) blocks. Six epochs were acquired in total (three activation and three rest).

\section{Scanning Parameters}

The data were acquired on a Siemens Magnetom Vision (Siemens, Erlangen, Germany) at $2 \mathrm{~T}$. Each BOLD-EPI volume scan consisted of 48 transverse slices (in-plane matrix $64 \times 64$; voxel size $3 \times 3 \times 3$ $\mathrm{mm}$; TE $=40 \mathrm{~ms} ; \mathrm{TR}=4.1 \mathrm{~s}$ ). Seventy-eight volume scans were collected during each cognitive and motor session, and 36 scans per visual session (epoch length was always 6 scans). A T1-weighted high-resolution MRI of the subject $(1 \times 1 \times 1.5 \mathrm{~mm}$ resolution) was acquired to facilitate anatomical localization of the functional data.

\section{Image Preprocessing}

Data preprocessing was carried out using SPM99 (Wellcome Department of Cognitive Neurology, London, UK; http:/www.fil.ion.ucl.ac.uk/spm). All functional volumes, independent of session or paradigm, were realigned to the first volume acquired (Friston et al., 1995) and a mean realigned volume was created. Sessions containing obvious movement artifacts (de- 
TABLE 2

Talairach coordinates

\section{Area}

Cluster size $(\mathrm{k})^{\mathrm{a}}$

Z score ${ }^{\mathrm{b}}$

Y

Right inferior parietal lobule Right inferior parietal lobule Right supramarginal gyrus

Left medial precentral gyrus (FEF)

Left lateral precentral gyrus

Left middle frontal gyrus

Left precuneus

Left superior parietal lobule

Left superior parietal Iobule

Right medial precentral gyrus (FEF)

Right superior frontal gyrus

Right superior frontal gyrus

Right superior parietal lobule

Right superior parietal lobule

Right superior parietal lobule

Right anterior precentral gyrus

Left middle frontal gyrus

Left middle frontal gyrus

Left middle frontal gyrus

Right lateral premotor cortex

Left supplementary motor area

Right inferior frontal gyrus

Right inferior frontal gyrus

Right superior temporal gyrus

Left inferior frontal gyrus

Left inferior frontal gyrus

Left insula

Right cerebellum, anterior lobe

Right cerebellum, anterior lobe

Right cerebellum, anterior lobe

Left tempero-occipital sulcus

Right inferior parietal lobule

Right inferior parietal lobule

Right inferior parietal lobule

Right middle frontal gyrus

Right middle frontal gyrus

Right middle frontal gyrus

Right anterior cingulate gyrus

Left inferior parietal lobule

Right middle temporal gyrus

Right superior temporal gyrus

Right middle occipital gyrus

Left precentral gyrus

Left middle frontal gyrus

Left superior temporal gyrus

Left inferior frontal gyrus

Right cerebellum

Right calcarine cortex (V1)

Right insula

Left cerebellum

Left anterior cingulate

Left inferior frontal gyrus

Left inferior frontal gyrus

Right insula

Left calcarine cortex (V1)

Right middle frontal gyrus

Left calcarine cortex (V1)

Left hemisphere, white matter.

Right superior frontal gyrus (a) Local maxima for the cognitive fixed-effects analysis

\begin{tabular}{|c|c|c|c|c|}
\hline \multirow[t]{3}{*}{888} & 9.27 & 50 & -30 & 44 \\
\hline & 8.63 & 34 & -44 & 44 \\
\hline & 7.95 & 66 & -24 & 40 \\
\hline \multirow[t]{3}{*}{1716} & 9.26 & -24 & 0 & 50 \\
\hline & 8.96 & -50 & 4 & 46 \\
\hline & 8.51 & -54 & 10 & 26 \\
\hline \multirow[t]{3}{*}{1812} & 9.17 & -10 & -66 & 44 \\
\hline & 8.86 & -42 & -36 & 50 \\
\hline & 8.77 & -18 & -62 & 64 \\
\hline \multirow[t]{3}{*}{776} & 9.17 & 26 & -4 & 58 \\
\hline & 8.70 & 20 & 6 & 60 \\
\hline & 7.68 & 12 & 14 & 66 \\
\hline \multirow[t]{3}{*}{876} & 8.99 & 20 & -66 & 58 \\
\hline & 8.74 & 26 & -70 & 50 \\
\hline & 8.42 & 22 & -58 & 62 \\
\hline 238 & 8.84 & 62 & 4 & 28 \\
\hline \multirow[t]{3}{*}{454} & 8.63 & -34 & 36 & 22 \\
\hline & 8.22 & -48 & 40 & 24 \\
\hline & 7.11 & -30 & 46 & 34 \\
\hline 85 & 8.39 & 56 & 0 & 46 \\
\hline 421 & 8.30 & -2 & 18 & 50 \\
\hline \multirow[t]{3}{*}{406} & 8.30 & 56 & 16 & -2 \\
\hline & 7.44 & 48 & 26 & -8 \\
\hline & 7.44 & -34 & 16 & 6 \\
\hline \multirow[t]{3}{*}{414} & 8.24 & -56 & 12 & 0 \\
\hline & 8.09 & -42 & 22 & -8 \\
\hline & 7.23 & -34 & 16 & 6 \\
\hline \multirow[t]{3}{*}{190} & 8.00 & 30 & -62 & -30 \\
\hline & 7.32 & 34 & -54 & -36 \\
\hline & 5.86 & 40 & -60 & -36 \\
\hline 41 & 7.96 & -52 & -52 & -18 \\
\hline \multirow[t]{3}{*}{44} & 7.92 & 68 & -38 & 24 \\
\hline & 5.48 & 68 & -30 & 32 \\
\hline & 5.33 & 64 & -44 & 34 \\
\hline \multirow[t]{3}{*}{311} & 7.91 & 44 & 40 & 32 \\
\hline & 7.81 & 44 & 42 & 20 \\
\hline & 7.80 & 34 & 36 & 18 \\
\hline 37 & 7.75 & 12 & 12 & 38 \\
\hline 80 & 7.67 & -62 & -36 & 32 \\
\hline 72 & 7.65 & 58 & -20 & -6 \\
\hline 67 & 7.62 & 48 & -32 & -2 \\
\hline 27 & 7.21 & 50 & -74 & 12 \\
\hline 11 & 6.99 & -54 & -8 & 14 \\
\hline 28 & 6.99 & -30 & 56 & 26 \\
\hline 43 & 6.96 & -54 & -40 & 4 \\
\hline 16 & 6.67 & -54 & 34 & 8 \\
\hline 16 & 6.58 & 36 & -38 & -42 \\
\hline 17 & 6.45 & 12 & -78 & 8 \\
\hline 11 & 6.39 & 40 & 6 & 0 \\
\hline 30 & 6.36 & -22 & -56 & -34 \\
\hline 9 & 6.35 & -12 & 16 & 32 \\
\hline \multirow[t]{2}{*}{23} & 6.02 & -36 & 58 & -2 \\
\hline & 5.53 & -32 & 62 & 4 \\
\hline 11 & 5.90 & 42 & 16 & 4 \\
\hline 17 & 5.80 & -6 & -72 & 20 \\
\hline 7 & 5.77 & 40 & 30 & 24 \\
\hline 6 & 5.50 & -4 & -9 & 0 \\
\hline 9 & 5.44 & -22 & 54 & 0 \\
\hline 5 & 5.24 & 16 & 38 & 54 \\
\hline
\end{tabular}


TABLE 2-Continued

Talairach coordinates

Area

Cluster size $(k)^{a}$

Z score ${ }^{b}$

Y

Z

(b) Local maxima for the cognitive random-effects analysis

Left superior parietal lobule

Left posterior postcentral gyrus

Left superior parietal lobule

Left medial precentral gyrus (FEF)

Left middle frontal gyrus

Left ventral precentral gyrus

Left superior parietal lobule

Left superior parietal lobule

Left superior parietal gyrus

Right inferior parietal lobule

Right inferior parietal lobule

Right supramarginal gyrus

Right superior parietal lobule

Right superior parietal lobule

Right superior parietal lobule

Right medial precentral gyrus (FEF)

Right superior frontal gyrus

Right superior frontal gyrus

Left precuneus

Right anterior precentral gyrus

Left middle frontal gyrus

Left middle frontal gyrus

Right cerebellum, anterior lobe

Right cerebellum, anterior lobe

Right inferior parietal lobule

Right middle temporal gyrus

Right lateral premotor cortex

Left inferior frontal gyrus

Left inferior frontal gyrus

Right inferior frontal gyrus

Left temporal-occipital sulcus

Left supplementary motor area (SMA)

Left supplementary motor area (SMA)

Left supplementary motor area (SMA)

Right anterior cingulate gyrus

Right middle frontal gyrus

Right middle frontal gyrus

Right middle frontal gyrus

Left inferior parietal lobule

Right middle frontal gyrus

Right superior temporal gyrus

Left middle frontal gyrus

Right superior temporal gyrus

Right inferior frontal gyrus

Left superior frontal gyrus

\begin{tabular}{|c|c|c|c|c|}
\hline \multirow[t]{3}{*}{690} & 8.13 & -40 & -36 & 48 \\
\hline & 7.44 & -48 & -28 & 40 \\
\hline & 7.28 & -30 & -48 & 42 \\
\hline \multirow[t]{3}{*}{1134} & 8.07 & -26 & -2 & 50 \\
\hline & 8.06 & -50 & 8 & 44 \\
\hline & 7.74 & -52 & 10 & 26 \\
\hline \multirow[t]{3}{*}{156} & 7.87 & -18 & -64 & 64 \\
\hline & 6.58 & -26 & -62 & 60 \\
\hline & 5.59 & -34 & -60 & 60 \\
\hline \multirow[t]{3}{*}{460} & 7.87 & 48 & -30 & 44 \\
\hline & 7.50 & 56 & -32 & 44 \\
\hline & 6.84 & 64 & -24 & 40 \\
\hline \multirow[t]{3}{*}{540} & 7.75 & 18 & -64 & 58 \\
\hline & 6.97 & 22 & -56 & 62 \\
\hline & 6.93 & 26 & -64 & 34 \\
\hline \multirow[t]{3}{*}{569} & 7.73 & 28 & -2 & 60 \\
\hline & 7.62 & 20 & 6 & 62 \\
\hline & 6.47 & 12 & 12 & 68 \\
\hline 157 & 7.72 & -8 & -66 & 42 \\
\hline 173 & 7.56 & 62 & 6 & 28 \\
\hline \multirow[t]{2}{*}{276} & 7.38 & -34 & 38 & 24 \\
\hline & 6.81 & -50 & 36 & 24 \\
\hline \multirow[t]{2}{*}{78} & 7.24 & 30 & -62 & -30 \\
\hline & 6.56 & 32 & -56 & -36 \\
\hline 22 & 7.03 & 68 & -38 & 24 \\
\hline 55 & 6.95 & 60 & -20 & -8 \\
\hline 41 & 6.94 & 54 & 2 & 44 \\
\hline \multirow[t]{2}{*}{139} & 6.92 & -42 & 22 & -8 \\
\hline & 6.80 & -56 & 12 & 0 \\
\hline 122 & 6.89 & 56 & 16 & -2 \\
\hline 18 & 6.77 & -52 & -52 & -18 \\
\hline \multirow[t]{3}{*}{201} & 6.71 & -2 & 18 & 48 \\
\hline & 6.66 & -6 & 10 & 54 \\
\hline & 6.64 & 0 & 16 & 58 \\
\hline 15 & 6.66 & 14 & 12 & 38 \\
\hline \multirow[t]{3}{*}{98} & 6.53 & 38 & 38 & 34 \\
\hline & 5.93 & 44 & 40 & 20 \\
\hline & 5.66 & 34 & 44 & 38 \\
\hline 33 & 6.26 & -64 & -38 & 26 \\
\hline 19 & 6.26 & 34 & 36 & 18 \\
\hline 26 & 6.01 & 46 & -30 & -4 \\
\hline 7 & 5.98 & -30 & 54 & 28 \\
\hline 16 & 5.90 & 54 & 18 & -20 \\
\hline 16 & 5.79 & 50 & 26 & -8 \\
\hline 7 & 5.73 & -10 & 12 & 70 \\
\hline
\end{tabular}

Note. FEF, frontal eye fields; V1, primary visual cortex; SMA, supplementary motor area.

a,b See footnotes to Table 1.

cided by two of the authors with previous experience of typical artifacts) were discarded at this stage: three motor sessions, two visual sessions, and three cognitive sessions were excluded in this manner. The subject's T1-weighted structural scan was coregistered to the mean functional volume, and the mean volume used to determine the parameters applied to all volumes during spatial normalization and resampling (Ashburner et al., 1997; Ashburner and Friston, 1999) to a stan- dard template (Evans et al., 1993). As the volume of brain sampled in each study was affected by the position of the subject within the scanner's field of view, we found that the extreme superior and inferior portions of the subject's brain were sparsely sampled. To address this, voxels not sampled in every session were eliminated during normalization. All functional volumes were then smoothed with a FWHM Gaussian kernel. Global changes in fMRI response from scan to 
TABLE 3

\begin{tabular}{|c|c|c|c|c|c|}
\hline Area & Cluster size (k) & Z score & $x$ & Y & Z \\
\hline \multicolumn{6}{|c|}{ (a) Local maxima for the visua fixed-effects analysis } \\
\hline Right calcarine cortex (V1) & 11002 & 9.72 & 14 & -86 & \\
\hline Right calcarine cortex (V1) & & 9.63 & 6 & -74 & -4 \\
\hline Left calcarine cortex (V1) & & 9.57 & -8 & -82 & 0 \\
\hline Right superior temporal gyrus & 80 & 7.96 & 42 & -28 & 18 \\
\hline Right precuneus & 54 & 7.93 & 8 & -80 & 44 \\
\hline Left lateral geniculate nucleus & 91 & 7.92 & -18 & -26 & -4 \\
\hline Right superior temporal gyrus & 51 & 7.81 & 70 & -32 & 14 \\
\hline Left inferior parietal lobule & 152 & 7.79 & -32 & -38 & 54 \\
\hline Left superior parietal lobule & 130 & 7.75 & -28 & -54 & 54 \\
\hline Left superior parietal lobule & & 6.66 & -20 & -52 & 54 \\
\hline Left superior parietal lobule & & 5.08 & -28 & -48 & 62 \\
\hline Left superior temporal gyrus & 32 & 7.60 & -56 & -42 & 16 \\
\hline Right lateral geniculate nucleus & 83 & 7.59 & 24 & -24 & 4 \\
\hline Right postcentral gyrus & 95 & 7.52 & 36 & -32 & 54 \\
\hline Left superior temporal gyrus & 31 & 7.17 & -42 & -28 & 20 \\
\hline Right lateral ventricle & 112 & 6.82 & 20 & -26 & 24 \\
\hline Left precuneus & 25 & 6.76 & -18 & -62 & 48 \\
\hline Right midbrain & 27 & 6.71 & 8 & -26 & -6 \\
\hline Right middle frontal gyrus & 22 & 6.58 & 60 & 2 & 38 \\
\hline Right inferior frontal gyrus & 26 & 6.46 & 56 & 36 & 12 \\
\hline Right inferior frontal gyrus & & 5.50 & 52 & 42 & 16 \\
\hline Left lateral ventricle & 58 & 6.30 & -16 & -22 & 26 \\
\hline Right precuneus & 12 & 6.24 & 28 & -62 & 48 \\
\hline Right cerebellum & 18 & 6.18 & 12 & -70 & -46 \\
\hline Right lateral ventricle & 11 & 6.18 & 4 & 12 & 12 \\
\hline Right parietal lobe, white matter & 7 & 6.18 & 36 & -58 & 64 \\
\hline Right supplementary motor area & 8 & 5.50 & 2 & 2 & 64 \\
\hline Right cerebellum & 6 & 5.49 & 32 & -72 & -30 \\
\hline Right fusiform gyrus & 5 & 5.48 & 48 & -50 & -16 \\
\hline Right temporal lobe, white matter & 6 & 5.40 & 34 & -40 & 10 \\
\hline Right superior parietal lobule & 5 & 5.20 & 40 & -52 & 54 \\
\hline
\end{tabular}

Talairach coordinates

(b) Local maxima for the visual random-effects analysis

Right calcarine cortex (V1)

Right extrastriate cortex

Right extrastriate cortex

Right precuneus

Right parieto-occipital fissure

Right superior temporal gyrus

Left superior parietal lobule

Left lateral geniculate nucleus

Left inferior parietal lobule

Right postcentral gyrus

Right lateral geniculate nucleus

Right superior temporal gyrus

Right inferior precentral gyrus

Left intraparietal sulcus

Left superior temporal gyrus

Right intraparietal sulcus

Left superior temporal gyrus

Right middle frontal gyrus

Right intraparietal sulcus

Left inferior occipital gyrus

\begin{tabular}{rrrrr}
8766 & 8.66 & 10 & -80 & -8 \\
& 8.59 & 24 & -96 & 16 \\
46 & 8.54 & 22 & -76 & -18 \\
& 7.48 & 8 & -78 & 44 \\
80 & 7.23 & 12 & -86 & 38 \\
37 & 6.68 & 42 & -28 & 18 \\
27 & 6.49 & -26 & -56 & 54 \\
47 & 6.21 & -16 & -28 & -4 \\
17 & 6.06 & -32 & -38 & 56 \\
21 & 6.04 & 34 & -32 & 52 \\
17 & 5.98 & 22 & -26 & 0 \\
6 & 5.96 & 70 & -34 & 12 \\
13 & 5.87 & 64 & 10 & 6 \\
9 & 5.86 & -20 & -70 & 34 \\
26 & 5.72 & -40 & -26 & 20 \\
7 & 5.71 & 32 & -70 & 26 \\
6 & 5.70 & -56 & -42 & 18 \\
8 & 5.57 & 58 & 4 & 38 \\
5 & 5.34 & 24 & -76 & 38 \\
\hline
\end{tabular}

Note. V1, primary visual cortex. 
a

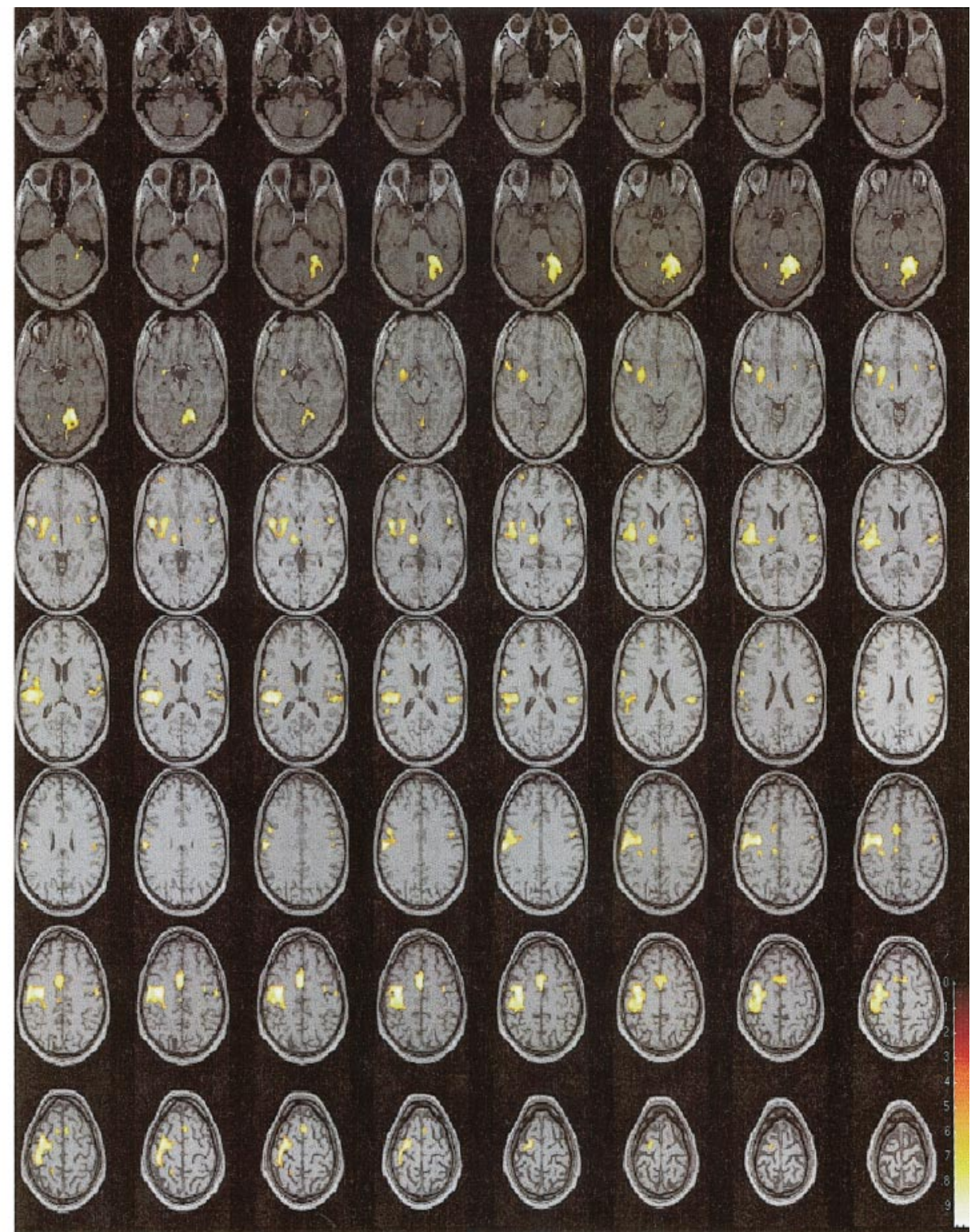

FIG. 5. Multisession analyses of the motor paradigm, analyzed using a fixed-effects model (a), extra-sum of squares $F$ test (b), and a random-effects model (c). Voxels surviving the statistical threshold are displayed on a coregistered structural scan of the subject to aid the identification of activated areas. Each transverse slice is $2 \mathrm{~mm}$ thick. The color bar represents statistical significance, with higher $\mathrm{Z}$ and $\mathrm{F}$ scores having a brighter color.

scan were removed by proportionally scaling each scan to have a common global mean voxel value.

\section{Theory and Implementation}

Statistical analysis was carried out using the general linear framework described by Worsley and Friston (1995). The sessions for each paradigm were modeled with a simple linear model for the data at each voxel:

$$
\mathrm{Y}_{\mathrm{ij}}=\gamma_{\mathrm{i}}+\alpha_{\mathrm{i}} \mathrm{f}(\mathrm{j})+\sum_{\mathrm{k}=1}^{\mathrm{K}} \beta_{\mathrm{ik}} \mathrm{g}_{\mathrm{k}}(\mathrm{j})+\epsilon_{\mathrm{ij}} .
$$

Here $Y_{i j}$ denotes the value of the voxel of scan $j(j=$ $1, \ldots, J)$ of session $\mathrm{i}(\mathrm{i}=1, \ldots, \mathrm{l}) . \gamma_{i}$ is the mean (block) effect for session i. $f(j)$ is a reference waveform, a function of the scan index within session which has the same form for all sessions. Here we shall use a simple "convolved boxcar" reference waveform (CBC), consisting of a boxcar function of zeros and ones representing the experimental time course, convolved with the expected hemodynamic response function. The parameter $\alpha_{i}$ is the amplitude of the CBC response for session i. Differences in the session response amplitudes $\alpha_{\mathrm{i}}$ constitute session-by-condition interac- 
b

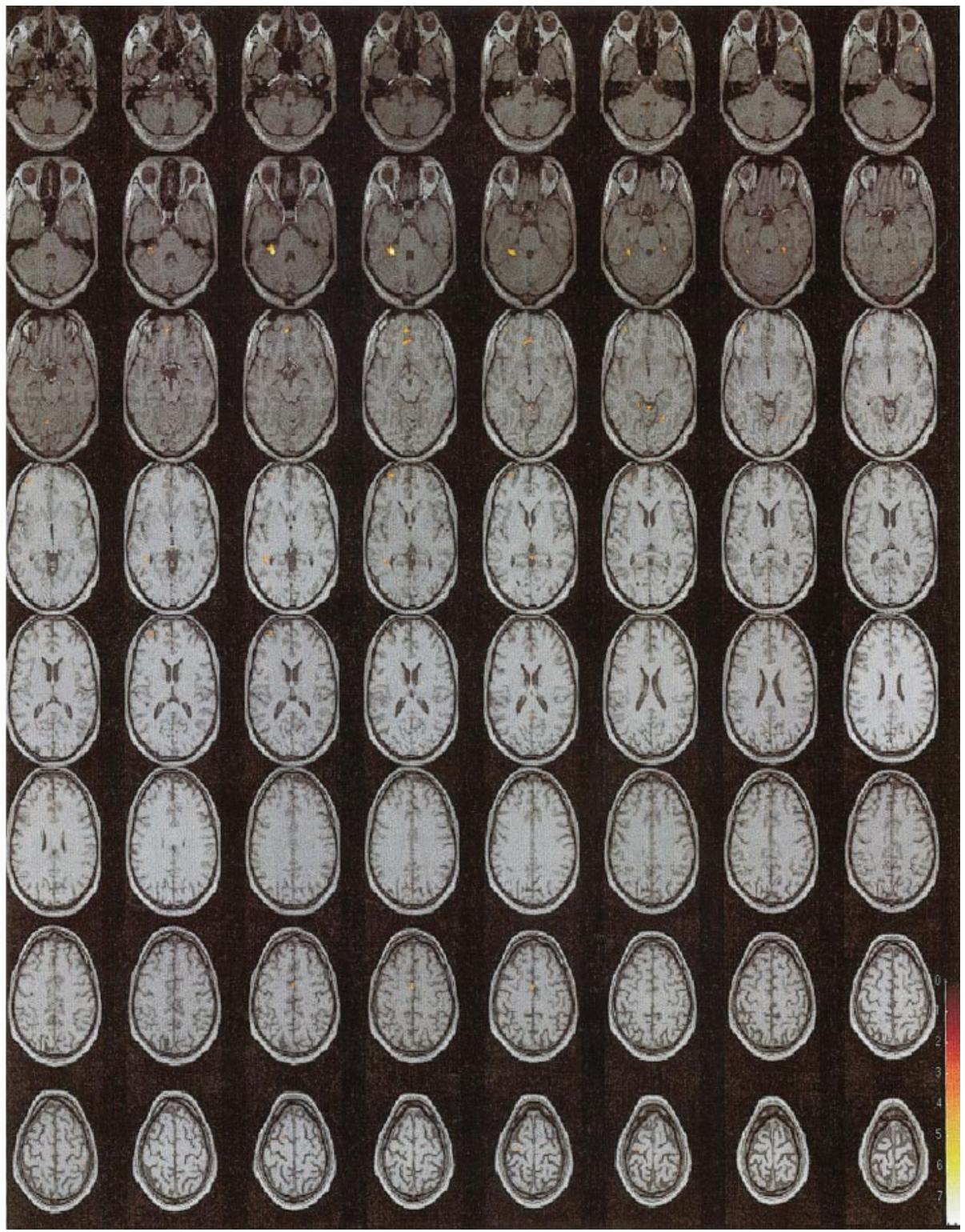

FIG. 5-Continued

tions. The additional reference functions $g_{k}(j)$ are a set of discrete cosine basis functions, effecting a simple "high-pass" filter, as described by Holmes et al. (1997), with cutoff (specified by K) set at twice the experimental period. We shall assume that this model fits, such that the residual errors $\left(\epsilon_{i j}\right)$ have zero mean and exhibit only short-term autocorrelation within session. In the following we shall refer to the CBC amplitudes $\alpha_{\mathrm{i}}$ simply as the response for session i.

\section{Individual-Session Analyses}

Each session was analyzed alone as a single fMRI session, as if it were the only session acquired, using a "standard" SPM analysis. The Groundhog Day effects aside, this enables a comparison of how the results of a single-session experiment can vary and illustrates why drawing conclusions about a subject from a single session can be dangerous. The model used is that of Eq. (1), but considering only a single session (i) at a time. The residual errors are assumed to be Normally distributed with variance $\sigma_{i(\epsilon))}^{2}$, estimated individually for each session. Temporal autocorrelation was dealt with using the method of Worsley and Friston (1995) by temporally smoothing the session time series with a Gaussian kernel of 6-s FWHM. The design matrix for each session is illustrated in Fig. 1A. A t statistic assessing the null hypothesis of zero response $\left(\alpha_{i}=0\right)$ was constructed for each voxel, giving an SPM \{t\} for 


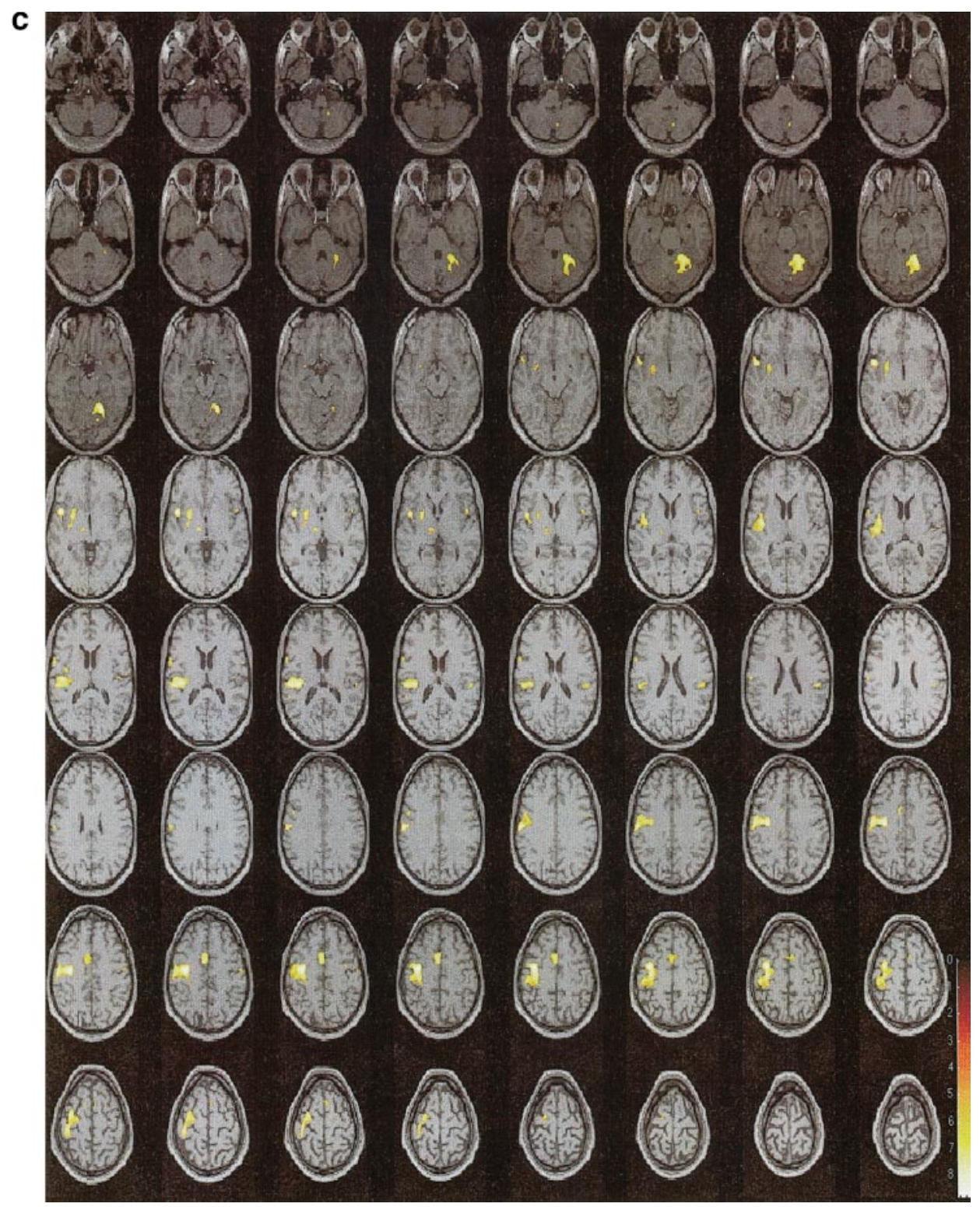

FIG. 5-Continued

each session indicating the significance of the response. For display, each session-specific SPM $\{t\}$ was transformed to an equivalent SPM $\{Z\}$ by probability integral transform. This was effected by replacing each $t$ value with the standard Normal ordinate with the same upper tail probability.

\section{MultipleSession Analyses-Session-by-Condition Interactions}

To assess whether there were significant sessionby-condition interactions, we compared the model of Eq. (1) for all I sessions (design matrix shown in Fig. 1B) with a reduced model in which the response was identical for all sessions $\left(\alpha_{\mathrm{i}}=\alpha^{\prime}, \mathrm{i}=1, \ldots, \mathrm{I}\right)$. Here we assume that the residual variance is identical across sessions, such that the residuals are Normally distributed with zero mean and variance $\sigma_{\epsilon}^{2}$. The additional variance modeled by the full model (including session-by-condition interactions) was compared with the residual variance using an extra sum-of-squares $F$ test (Draper and Smith, 1981), modified to account for temporally autocorrelated residuals using the method of Worsley and Friston (1995). The resulting SPM $\{F\}$ identifies voxels that display significant session-by-condition interactions. 


\section{Multiple-Session Analyses}

If there are substantial differences in response from session to session a single-session experiment is inadequate if one wishes to examine a subject's response to experimental stimuli in general, and so a multiplesession experiment is necessitated.

\section{Multiple-Session Analyses-Fixed-Effects Model}

Given a multiple-session data set, modeled with Eq. (1) (design matrix shown in Fig. 1B), a fixed-effects analysis proceeds by assuming that the session-specific responses $\alpha_{\mathrm{i}}$ themselves are of interest. The residual errors $\epsilon_{i j}$ are assumed Normally distributed with zero mean and constant variance $\sigma_{\epsilon}^{2}$. Evidence of a response across sessions can be tested by examining $\bar{\alpha}_{\text {., the }}$ average of the I session-specific responses

$$
\bar{\alpha} .=\sum_{\mathrm{i}=1}^{\mathrm{I}} \mathrm{a}_{\mathrm{i}} .
$$

Again, short-term temporal autocorrelation in the errors were handled using the method of Worsley and Friston (1995), temporally smoothing each session time series with a Gaussian kernel of 6-s FWHM.

However, since the session-specific responses are considered fixed, only one component of variance is accounted for (the residual error variance $\sigma_{\epsilon}^{2}$ ), and inference from the resulting SPM $\{t\}$ is limited to the average response for the observed sessions. As such, this analysis is sensitive to large effects in a small number of sessions.

\section{Multiple-Session Analyses-Random-Effects Model}

To extend inference beyond the particular sessions acquired, we must recognize that these sessions are merely a sample of possible sessions, each of which would have its own response $\alpha_{\mathrm{i}}$. Thus, we regard the $\alpha_{\mathrm{i}}$ of Eq. (1) as random effects, accepting that the response amplitudes $\alpha_{\mathrm{i}}$ for the sessions under consideration are merely one sample from the (hypothetical) distribution of response amplitudes for a session chosen at random. A simple second-level (between-session) model would be

$$
\alpha_{\mathrm{i}}=\alpha+\epsilon_{\mathrm{i}},
$$

where the $\alpha_{\mathrm{i}}$ are from Eq. (1) (the within-session model), and the between-session errors $\epsilon_{\mathrm{i}}$ have zero mean and variance $\sigma_{\alpha}^{2}$ and can be considered independent. Thus, the random-effects model has two components of variance, between session, $\sigma_{\alpha}^{2}$, and within session (residual), $\sigma_{\epsilon}^{2}$. Using this model we can consider inference regarding $\alpha$, the underlying average re sponse across all possible sessions.
In general, analysis of such random-effects models can be difficult (Searle and Casella, 1992). However, the simple models considered here are balanced (the models for each session are exactly the same) and separable (the only common parameter across sessions is the intrasession (residual) variance $\sigma_{\epsilon}^{2}$, assumed constant for all sessions). This permits a simple "summary statistic" approach (Frison and Pocock, 1992). Such an approach was first described for neuroimaging data by Worsley et al. (1992), and its importance subsequently highlighted by Holmes et al. (1998), who describe the implementation (in SPM) used here. In essence, the model of Eq. (1) is fitted to yield estimates $\hat{\alpha}_{i}$ of the response amplitude $\alpha_{i}$ at each voxel for each session. The variance of the estimated response amplitudes $\hat{\alpha}_{i}$ across sessions incorporates both within- $\left(\sigma_{\epsilon}^{2}\right)$ and between-session variability $\left(\sigma_{\alpha}^{2}\right)$ in the appropriate proportions to assess the significance of the overall subject activation effect $\alpha$ (Frison and Pocock, 1992). Thus, each session data set is surmised by a single contrast image whose voxel values are the fitted response amplitudes. These contrast images can then be assessed at the intersession level for a significant average effect, with inference extending to the subject in general (under similar experimental conditions) rather than just the particular sessions acquired.

To conduct a parametric analysis, it remains to choose a specific model for the between-session errors $\epsilon_{i}$. In the absence of any evidence (yet) to suggest otherwise, consider a simple Normal model

$$
\alpha_{\mathrm{i}}=\alpha+\epsilon_{\mathrm{i}}, \quad \epsilon_{\mathrm{i}} \sim \mathrm{N}\left(0, \sigma_{\alpha}^{2}\right) .
$$

Our approach here is pragmatic: we know nothing about $\epsilon_{\mathrm{i}}$ 's distribution. Our assumption of Normality allows us to introduce random effects analyses simply and logically as an extension of the parametric statistical tests used by SPM. We will discuss the validity of this assumption in the discussion.

With the models of Eqs. (1) and (3), the randomeffects analysis can be effected as a simple one-sample t test on the contrast images, yielding an SPM $\{t\}$.

\section{RESULTS}

All tabular data referenced in the results section can be accessed at http://www.fil.ion.ucl.ac.uk/ davem. The coordinate system used is that of the space defined by Talairach and Tournoux (1988).

\section{Individual Session Results}

Figures 2, 3, and 4 show sagittal maximum intensity projections (MIPs) per session for the motor, cognitive, and visual tasks, respectively. Each SPM $\{Z\}$ MIP shows voxels that survive a threshold of $P<0.05$, corrected for multiple comparisons. 
a

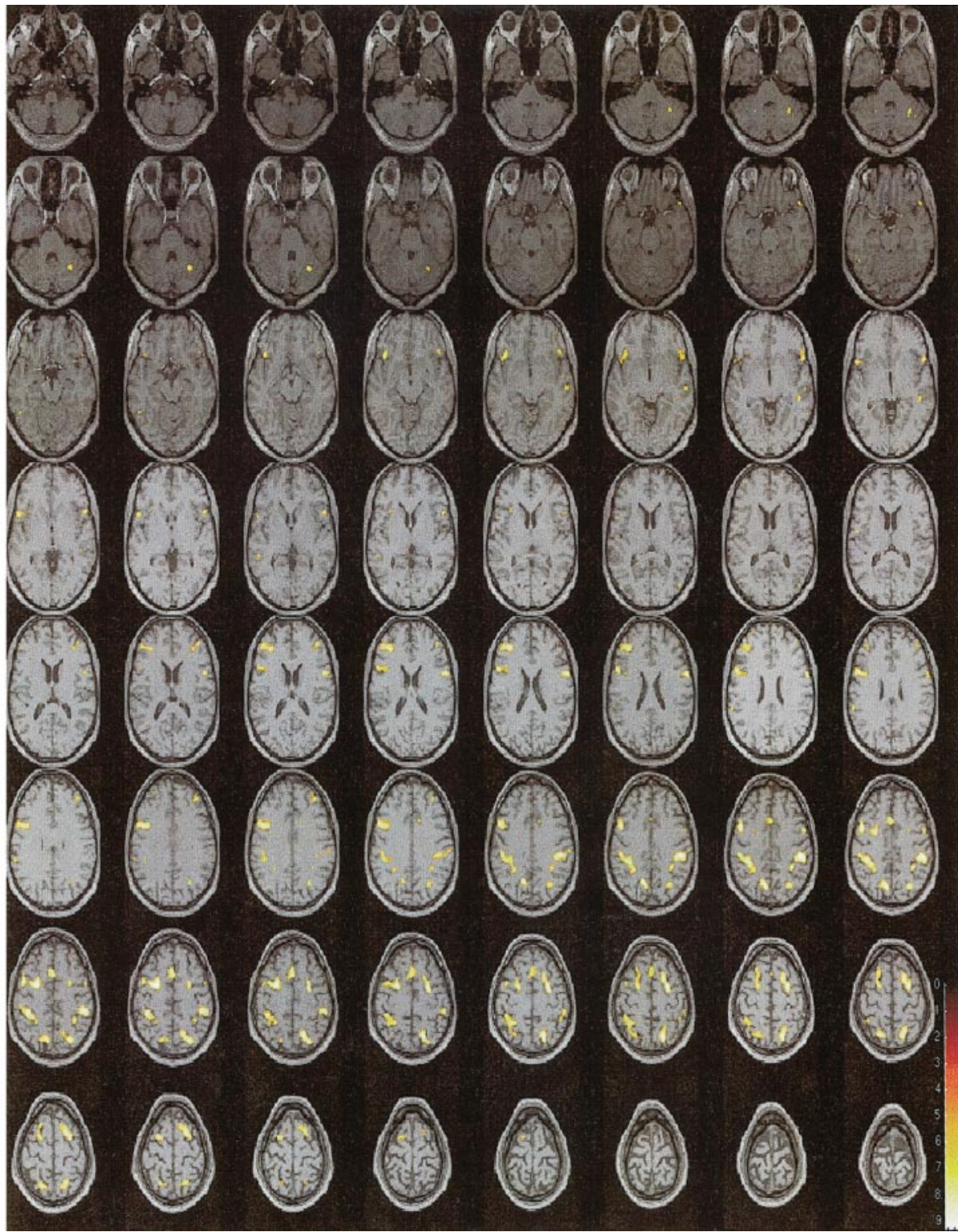

FIG. 6. Multisession analyses of the cognitive paradigm, analyzed using a fixed-effects model (a), extra-sum of squares $F$ test (b), and a random-effects model (c). Voxels surviving the statistical threshold are displayed on a coregistered structural scan of the subject to aid the identification of activated areas. Each transverse slice is $2 \mathrm{~mm}$ thick. The color bar represents statistical significance, with higher $\mathrm{Z}$ and $\mathrm{F}$ scores having a brighter color.

It is immediately obvious that the pattern of activated voxels varies widely between repeated single sessions in our subject. While a grossly homogeneous pattern is evident across single-session MIPS of the same paradigm, the spatial distribution of voxels in each MIP is highly variable. Even though striking similarity is evident between certain data sets (e.g., visual sessions 10 and 12, Fig. 4), a large number of sessions from all three paradigms display no significantly activated voxels (e.g., visual sessions 4 and 30 ). The differences are best exemplified by comparing the SPM $\{Z\}$ of motor session 1 (Fig. 2), which contains 1076 voxels above threshold, and motor session 33, which contains only 5. Results from the cognitive paradigm (Fig. 3) are broadly similar: while the spatial distribution of voxels between MIPs is more comparable than in the motor and visual paradigms, a large number of sessions contain no significantly activated voxels at the chosen threshold.

MIPs are binary statistical images, in which voxels are classified as "active" or "inactive" according to accepted but arbitrary statistical thresholds (for discussions of this issue, see Poline et al., 1996; Genovese et al., 1997; Noll et al., 1997; Cohen and DuBois, 1999; Tegeler et al., 1999). In any of the MIPs of Figs. 2, 3, and 4, a voxel i could have very different $\alpha_{i}$ 's between 


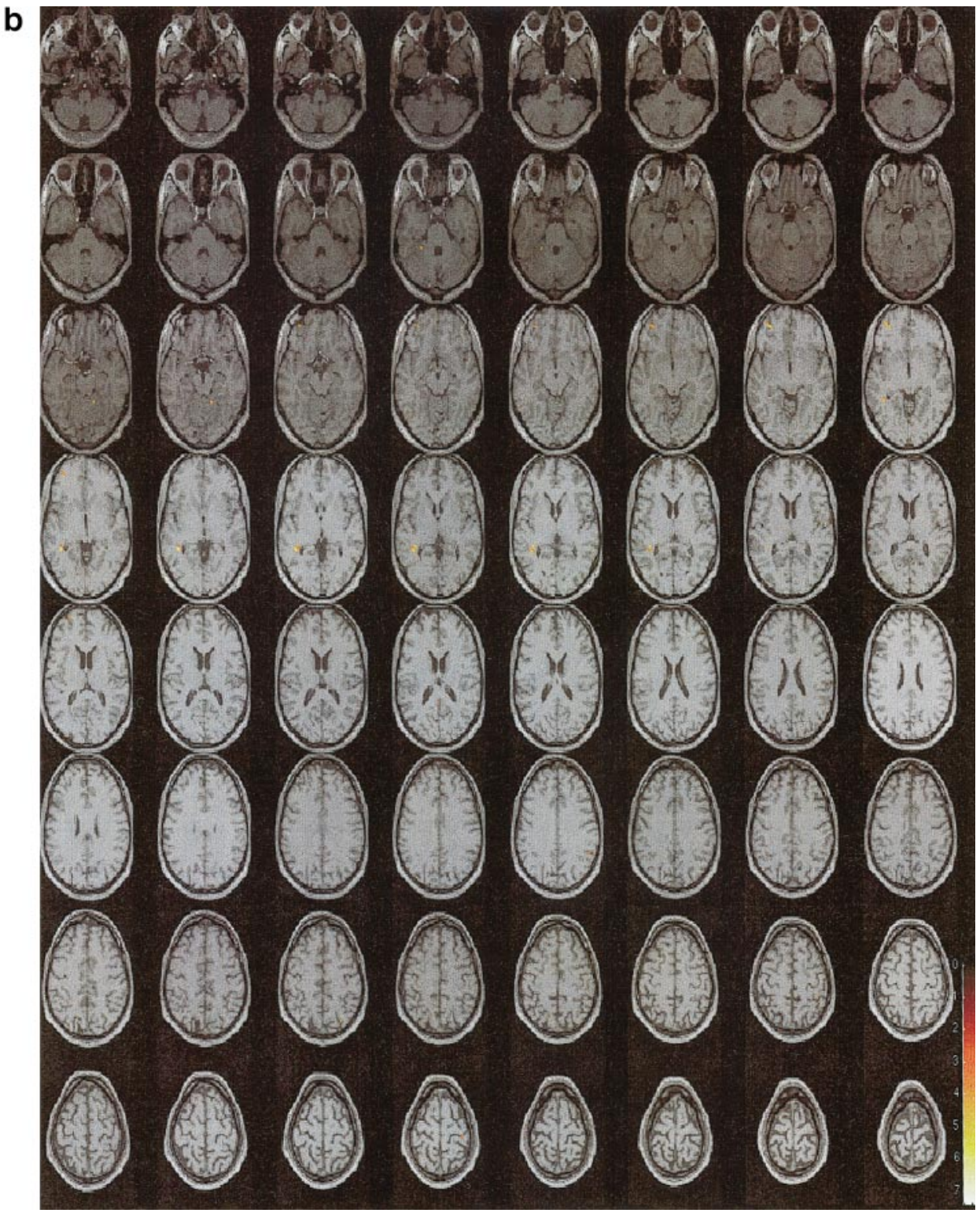

FIG. 6-Continued

sessions, yet still pass the threshold and appear to be consistently activated.

\section{Multiple-Session Analyses}

Figures 5, 6, and 7 show the results of the motor, cognitive, and visual multiple-session analyses, respectively. As noted above, merely examining thresholded statistical maps is perhaps not the best way to examine similarities between sessions. Our use of the ESS-F test allowed us to examine which voxels showed statistically significant variability across all sessions for our single subject (Figs. 5b, 6b, and 7b). If a single session typifies our subject's response, there should be few session-bycondition interactions, and thus the SPM $\{\mathbb{F}\}$ maps from each analysis should display relatively few voxels.
By specifically examining the variability of session-bycondition interactions, we implicitly limit our analysis to voxels that are activated on at least one session by the task. Noise that has a truly random expression over time is unlikely to be modeled sufficiently well by each session's regressor of interest; however, task-correlated noise, such as movement, will still present a problem.

\section{ESS $\{F\}$ Analyses}

Figures $5 b, 6 b$, and $7 b$ show the results of each multisession ESS-F test. These SPMs were thresholded at $\mathrm{P}<0.05$ corrected as for the fixed-effects SPM $\{Z\}$ 's, reflecting that we did not have any a priori hypotheses concerning where we expected to see greater variability. An important point to note at this 


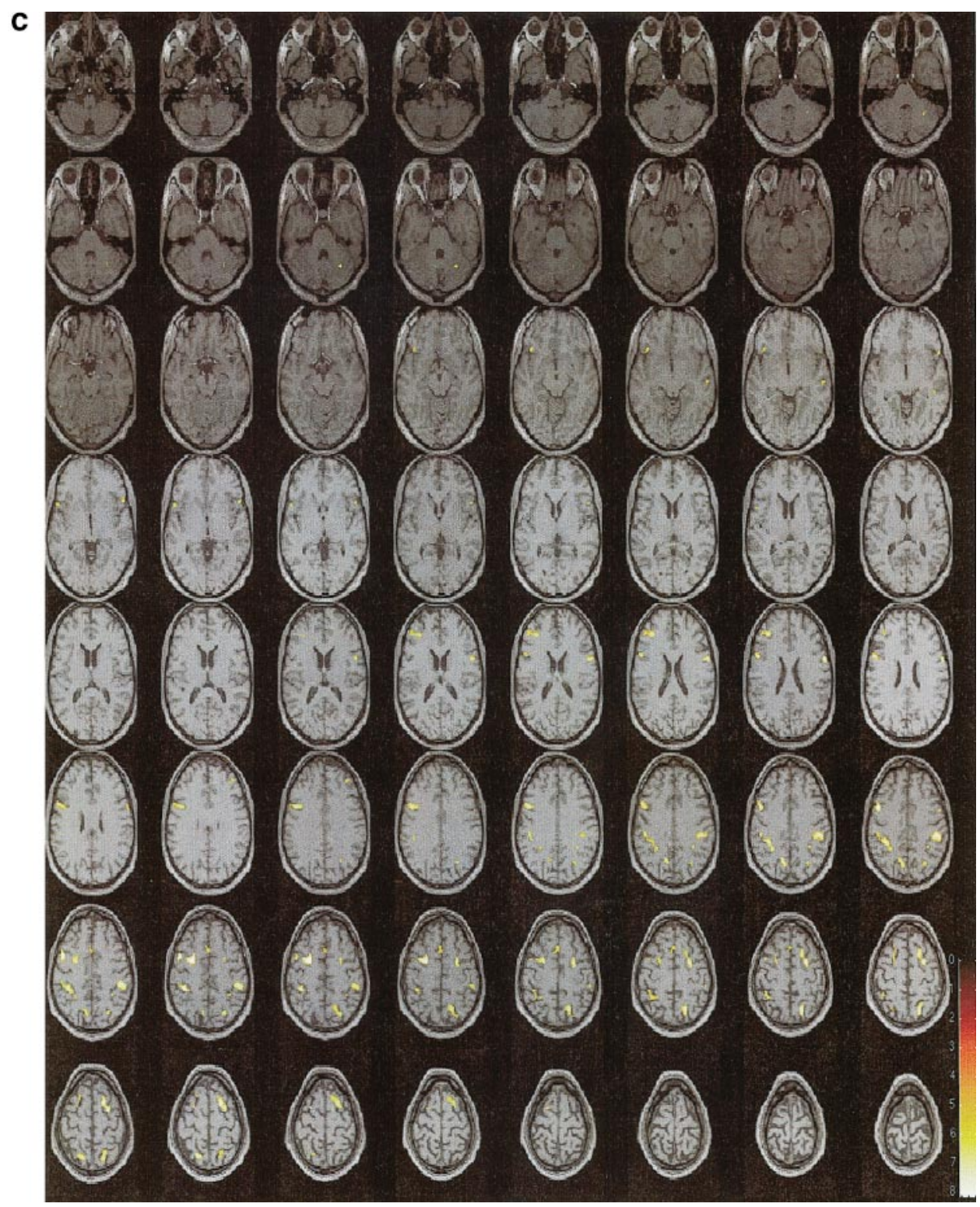

FIG. 6-Continued

stage is that the ESS-F test is free of any constraints about the direction of activation effects observed. As such, although our main concern was to examine the variability of activation effects, each SPM $\{$ F $\}$ also contains voxels that had highly variable deactivations. In the interest of parsimony, these results will not be discussed here.

Somewhat surprisingly, each fixed-effects SPM $\{Z\}$ did not display a high degree of overlap with its corresponding SPM $\{\mathbb{F}\}$. This is because each SPM $\{\mathbb{F}\}$ identifies voxels that show high variability, even if they are not classified as activated on average. Reflecting this fact, the area displaying the highest degree of variability in signal intensity between sessions in the motor paradigm (Fig. 5b) is located within the white matter of the temporal lobe $(-28,-42,-28, F=7.88)$-an area which does not appear on the fixed-effect SPM $\{$ \} map (Fig. 5a). A similar area is observed in the cognitive paradigm's SPM $\{F\}(-38,-40,6, F=7.40$; Fig. 6b); again, this area is not present on the fixed-effects SPM $\{$ \} (Fig. 6a). There was some overlap between voxels which displayed significant variability in each SPM $\{F\}$ and the corresponding fixed-effects SPM $\{Z$ : for example, posterior SMA $(-2,-8,52 ; \mathrm{F}=4.67)$, ipsilateral cerebellum $(26,-38,-22 ; \mathrm{F}=5.68)$, and contralateral precentral gyrus $(-26,-18,70 ; \mathrm{F}=$ 4.66). These voxels were typically located at the edge of a larger cluster of activated voxels. The variability seen may reflect subtle differences in the areal extent of activations at the periphery of large clusters. 


\section{Fixed-Effects Analyses}

The fixed-effects analyses of all three tasks (Figs. $5 a, 6 a$, and 7a) displayed areas of activation concordant with previous studies employing a similar task. A number of $\mathrm{fMRI}$ studies have used finger-tapping as a stereotypical motor task (e.g., Rao et al., 1993), and we found similar results (Table 1a), including the contralateral SM 1 (Talairach coord. -38, - 10, 52 ; $Z$ score $=9.77)$, the anteri or lobe of the ipsilateral cerebellum $(20,-54,-18 ; Z=9.50)$, the SMA $(-2$, $-2,52 ; Z=9.39)$, the contralateral thalamus $(-12$, $-18,2 ; Z=8.84)$, and the ipsilateral premotor cortex $(38,-8,50 ; Z=8.80)$. It is notable that the SPM $\{Z\}$ also contains areas not previously reported as activated by a simple externally paced finger-tapping paradigm, such as the right inferior parietal lobule $(50,-28,24 ; Z=8.54)$. This is not surprising, as a single $\alpha_{i}$ of sufficient magnitude may be adequate for any voxel to pass the average significance threshold over sessions and so appear on the multisession fixed-effects SPM $\{Z\}$. If the fixed-effects SPM $\{Z\}$ is viewed in isolation, it is impossible to know if these are "true" activated voxels which have not been reported in previous studies due to a lack of sensitivity or areas which display an activation effect significantly large to appear in the multisession fixed-effects maps, yet are not consistently activated across sessions.

Similar patterns of results were observed upon inspection of the multisession fixed-effects SPM $\{Z\}$ 's from the cognitive and visual paradigms (Figs. 6a and 7a). Although less is known about the functional neuroanatomy of paced random-number generation, we found areas similar to a previous study (Table 2a; J ahanshahi et al., submitted for publication). In common with J ahanshahi and colleagues, we found activation in the anterior cingulate cortex, but again noticed discrepancies between our results and theirs, e.g., our finding of bilateral cal carine cortex $(12,-78$, $8, Z=6.45$ and $-6,-72,20, Z=5.80)$ and left SMA activation $(-2,18,50, Z=8.30)$. Similarly, our visual paradigm activated, as expected, striate and extrastriate areas around the calacarine sulcus (Fig. $7 a$, Table 3a), including bilateral V1 $(14,-86,2, \mathrm{Z}=$ 9.72 and $-8,-82,0, Z=9.57)$, in common with studies employing a comparable stimulus (e.g., Kwong et al., 1992). However, as with the other paradigms, a number of areas not previously implicated in the functional neuroanatomy of this task were activated (e.g., the right SMA: 2, 2, 64, Z = 5.50).

Clearly, these effects beg closer scrutiny. If we wish to examine repeated trials of the same activation paradigm within a particular subject, it is necessary to define variability within the same subject. The fixedeffects SPM $\{$ \}'s tell us where voxels are active on average across the observed sessions. If we wish to examine the generality of a single fMRI session, the variability of each voxel across all sessions must be addressed.

\section{Random-Effects Analyses}

Figures $5 c, 6 c$, and $7 c$ show random-effects analyses of each multisession data set. These SPM $\{Z\}$ 's have been weighted by both between-session and withinsession variances of each data set. U pon visual inspection, the random-effects SPM $\{Z\}$ 's resemble a "cleaned-up" version of the fixed-effects SPM $\{Z\}$ 's, and each paradigm's pattern of results is now more in concordance with previous studies. There are still, however, areas within the random-effects SPM $\{Z\}$ that one would not expect, a priori, to be involved in the functional neuroanatomy of each task (Tables 1b, 2b, and 3b). For example, the motor random-effects SPM ZZ\} (Fig. 5c) displays prominent bilateral auditory cortex activation $(-42,-28,18, Z=8.14$ and $48,-18,14, Z=6.16)$. We did not expect this, as pacing tones were played during both rest and activation epochs during each motor session. This result may reflect attentional modulation of auditory areas (Woodruff et al., 1996; Grady et al., 1997), as the tones' salience was different between the rest and the activation conditions. The neurobiological explanation for this result need not concern us here: it is sufficient to recognize that we did not predict this pattern of activation. If we had access to only a single session from our subject, we would have been suspicious about their true nature. Even a multisession fixed effects would not have helped: we would not be able to identify if the activation was driven by a small number of sessions only or was indeed a true positive. This reasoning demonstrates that multiple scanning sessions analyzed with an appropriate statistical model can reduce ambiguous interpretations.

The majority of voxels present in both the fixedeffects SPM $\{Z\}$ and SPM $\{F\}$ do not appear in the random-effects SPM $\{Z\}$ 's. Properly accounting for between-session variance means that these voxels no longer survive a threshold of $\mathrm{P}<0.05$, corrected for multiple comparisons. This demonstrates that combining multiple sampling of sessions with a statistical model with more than one component of variance correctly accounts for even small session-by-condition interactions.

Figures 8 and 9 show voxels that typify different patterns of behavior across sessions, using the motor paradigm as an example. Figures $8 \mathrm{~A}$ and $9 \mathrm{~A}$ show a voxel in posterior SMA $(-2,-8,-52)$ which survives a threshold of $\mathrm{P}<0.05$, corrected for multiple comparisons, in our multisession fixed-effects analysis. However, this voxel displays significant session-by-condition interactions (as seen by its appearance on the ESS-F map) and thus fails to survive correction when a 


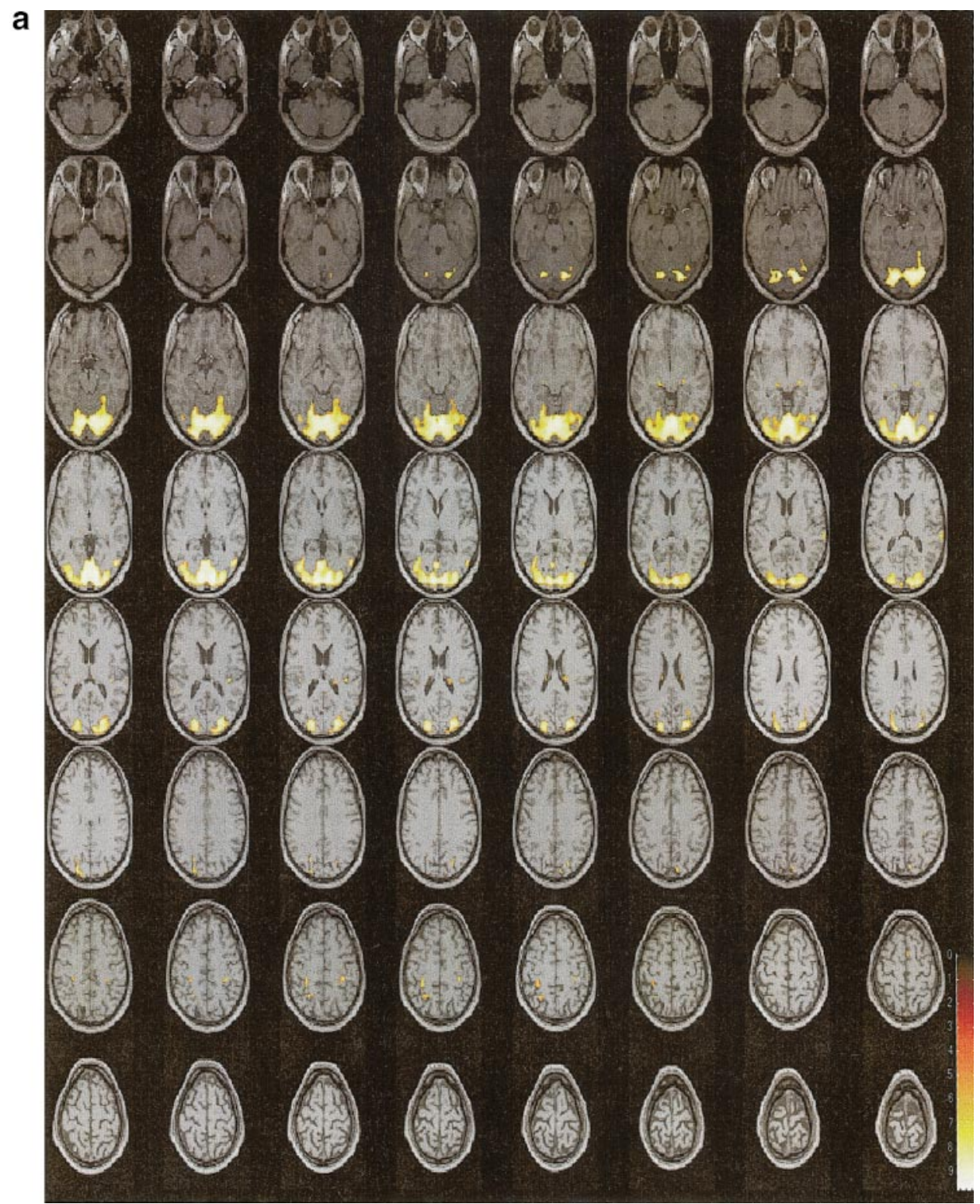

FIG. 7. Multisession analyses of the visual paradigm, analyzed using a fixed-effects model (a), extra-sum of squares $F$ test (b), and a random-effects model (c). Voxels surviving the statistical threshold are displayed on a coregistered structural scan of the subject to aid the identification of activated areas. Each transverse slice is $2 \mathrm{~mm}$ thick. The color bar represents statistical significance, with higher $\mathrm{Z}$ and $\mathrm{F}$ scores having a brighter color.

random-effects model is used. This voxel is an excellent example of variability in "active" voxels. When one examines its parameter estimates by session (Fig. 9A), it is striking how stable it appears over some sessions (for example, sessions 15 to 18) and yet how variable its behavior is over all sessions. The histogram of parameter estimates in Fig. 9A shows that although only one session has a parameter estimate of greater than 1.5, this can still weigh the average activation effect over all sessions. When the variability of responses over sessions is addressed in the random-effects analysis, the voxel loses significance.
The voxel in left primary motor cortex $(-36,-10,52)$ displayed in Figs. 8B and 9B typifies voxels that survive statistical thresholds in both fixed- and randomeffects analyses. This voxel shows remarkably similar parameter estimates over all sessions (Fig. 9B). The voxel in Figs. $8 \mathrm{C}$ and $9 \mathrm{C}$ is one that, although not significantly variable (not shown on the ESS $\{$ \} map in Fig. 8C), does not survive correction when a randomeffects model is used.

Voxels within each SPM $\{\mathrm{F}\}$ can be thought of as belonging to various classes: those which are not activated by each paradigm, but display high vari- 


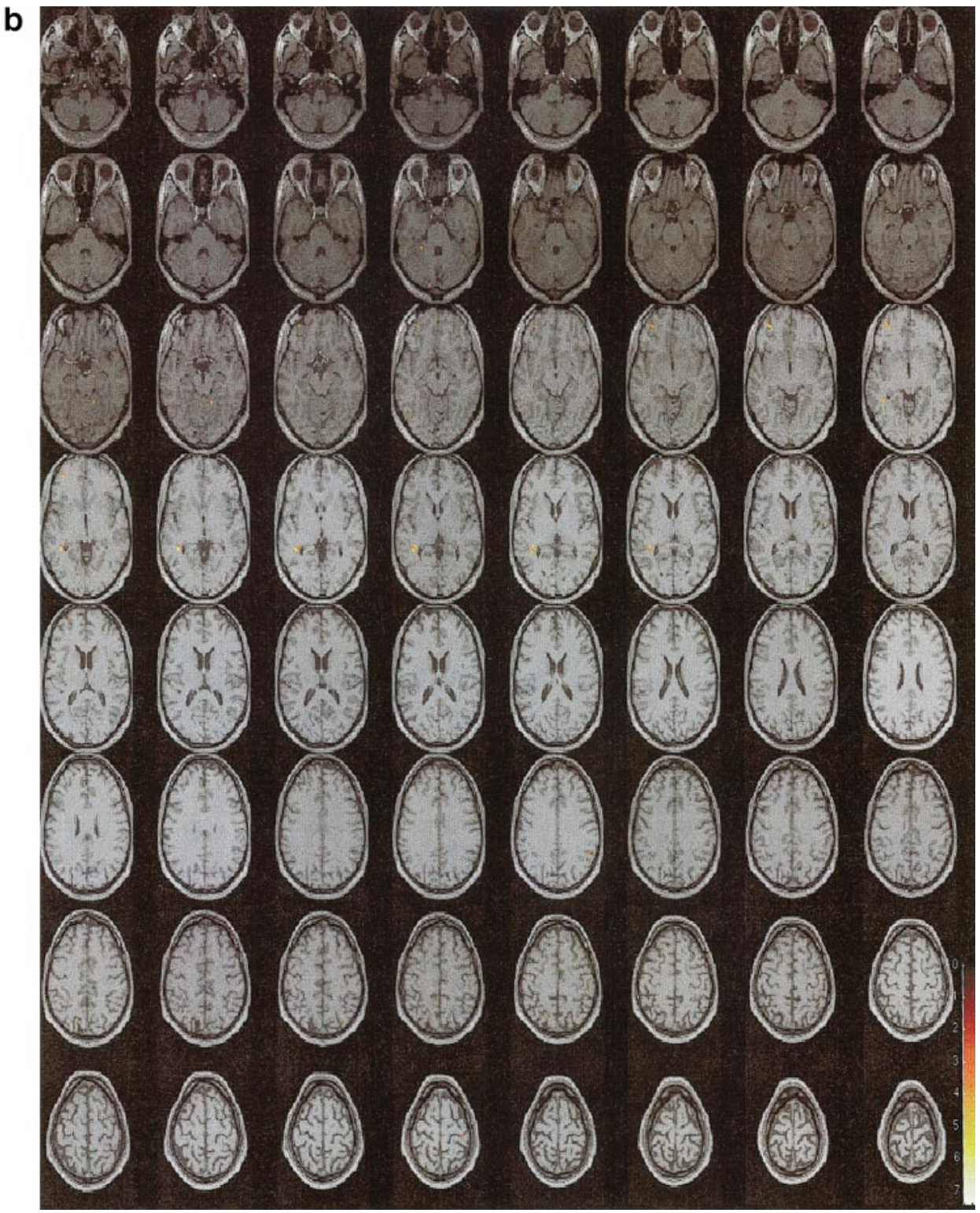

FIG. 7-Continued

ability of their parameter estimates (Figs. 8D and 9D); "true" active or deactivated voxels, surviving both fixed- and random-effects definitions of variability (Figs. 8B and 9B); voxels which are significant at a fixed-effects level but are significantly variable and do not survive correction for between-session variance (Figs. 8A and 9A); and voxels which, while not surviving a random-effects analysis, are not significantly variable as defined by the ESS $\{F\}$ map (Figs. 8C and 9C).

\section{DISCUSSION}

The generality of any experimental result is an issue which confronts all researchers, independent of exper- imental discipline (Abelson, 1995). The results of any isolated experiment are always open to contamination, and $\mathrm{fMRI}$ is no exception. As fMRI is an ideal experimental technique to examine questions that require serial scanning sessions, there have been a number of previous studies that sought to examine the reproducibility of fMRI data. Researchers have examined similar activation paradigms across laboratories (Casey et al., 1998), imaging modalities (Ojemann et al., 1998), and sessions (Le et al., 1997; Noll et al., 1997; Rombouts et al., 1998; Cohen et al., 1999). These studies sought to characterize the reproducibility of fMRI data and so tried to ensure that each session was carried out similarly to those preceding it. 


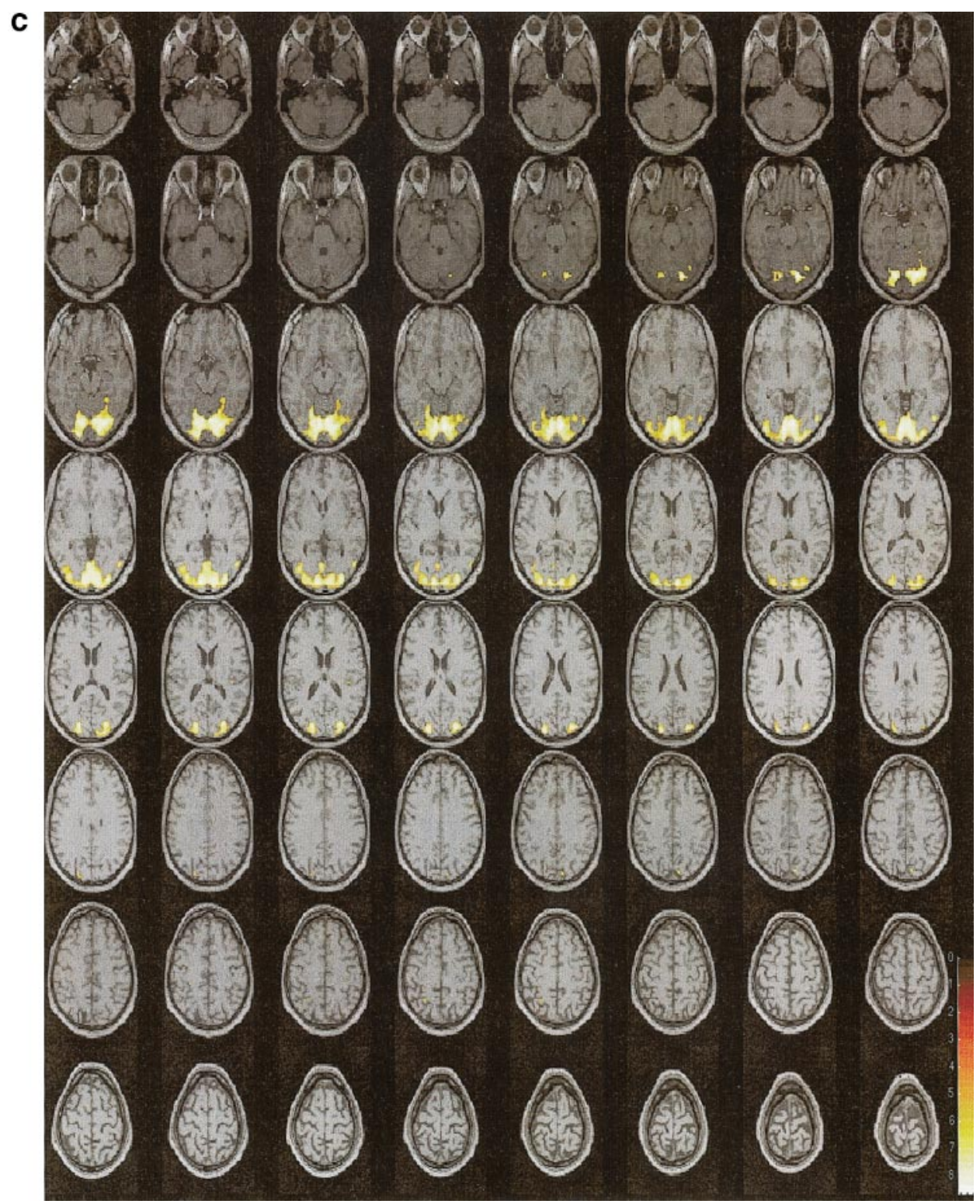

FIG. 7-Continued

Examining the reproducibility of $\mathrm{FMRI}$ data is an important question, but our own question was subtly different: we chose to examine how well a singlesession data set from a single subject typified the subject's response across multiple sessions, using a variety of activation paradigms. By examining the variability in the magnitude of activation effects across a large number of sessions we accepted that each session would be different. Indeed, it was exactly this between-session variability that we wished to quantify.

We found that significant session-by-condition interactions occurred in each of the multisession data sets that we examined, as illustrated by the respective ESS SPM $\{\mathbb{F}\}$ 's. Our results are evidence of the influence of session context on the results of any individual session and show the potential danger of drawing general conclusions from an individual session analyzed in isolation with nothing known about reproducibility. If one samples more sessions, each successive session acquired facilitates a better estimation of between-session variance, thereby increasing power to detect the underlying response.

\section{Differences in the Generality of Different Activation Paradigms}

We chose to examine different activation paradigms to ensure that the results of our study would not be limited to a single class of activation task. The majority 

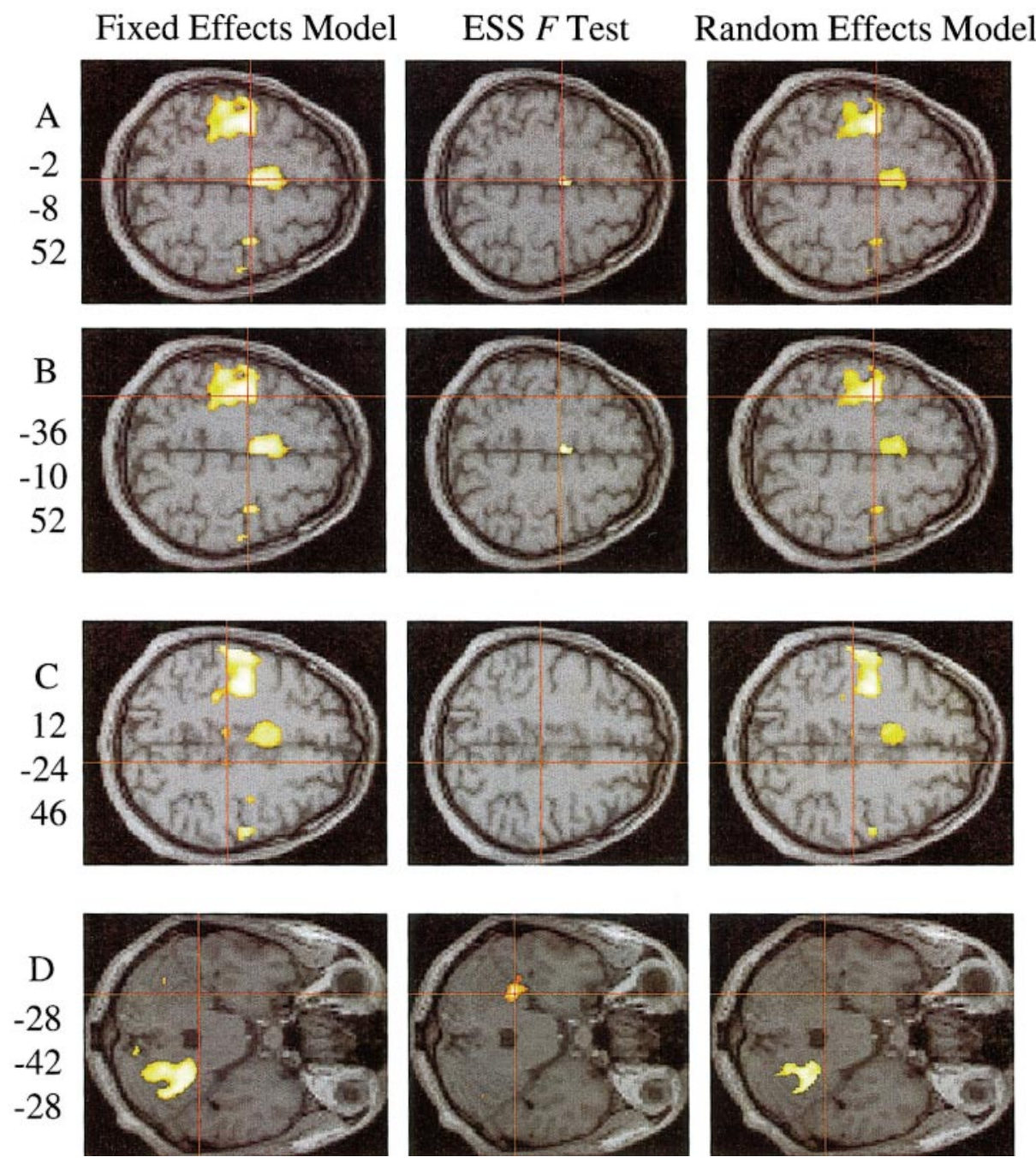

FIG. 8. Examples of voxels from the multisession motor analyses that typify different kinds of statistical significance. Voxel A is significant at a fixed-effects level, but variable enough to not appear in a random-effects analysis. Voxel B is significant at both a fixed- and a random-effects level and does not display significant session-by-condition interaction terms (it does not appear in the ESS-F). Voxel C is significant at a fixed-effects level and is not significantly variable across sessions, but does not possess a random-effects level of significance. Voxel D has significance at neither fixed- nor random-effects level, but is significantly variable between sessions to appear in the ESS-F.

of work examining repeatability in fMRI has employed simple visual or motor paradigms, though there has been limited use of "higher" cognitive activation paradigms (Yetkin et al., 1996; N oll et al., 1997; Casey et al., 1998). Our initial expectations were that the visual task would prove to have the fewest session-by-condition interactions and the cognitive task the most. While we did not compare the number or magnitude of voxels in each paradigm's SPM $\{F\}$, we were initially surprised to note that the visual SPM $\{\mathrm{F}\}$ appeared to have prominent bilateral areas of high variability in primary visual cortex (Fig. 7b), while the cognitive SPM $\{\mathbb{F}\}$ contained few voxels which overlapped with areas activated consistently by the activation task itself. As mentioned above, the visual activations were located in areas not activated on average by the task itself, but it is still surprising to find them in such close proximity to primary visual cortex. We suggest that slight differences in visual field coverage by our visual stimulus may have caused these effects, producing a variable rim around a core of visual cortex that was consistently stimulated across sessions. A further possibility is that these results reflect the high concentration of venules in the microvasculature of visual cortex (Marinković et al., 1995), which may cause a higher variability in its response to afferent stimulation.

\section{Sources of Session-by-Condition Interactions}

We did not attempt to systematically assess the relative magnitudes of different sources of variance on session reproducibility, as in some previous studies (e.g., Noll et al., 1997). We acknowledge, however, that identifying the sources of intersession variance 

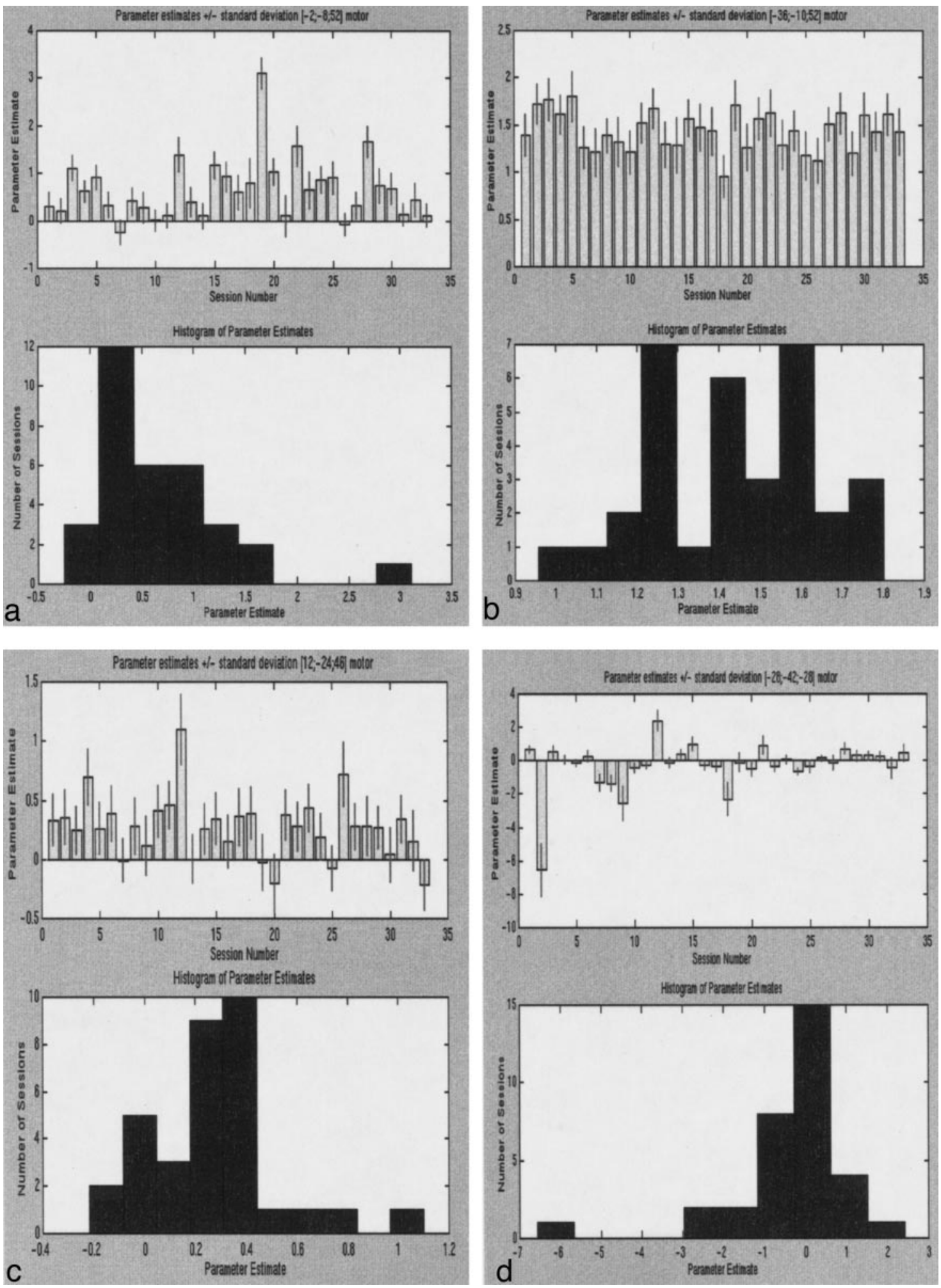

FIG. 9. Session-by-session plots of the parameter estimates $\left(\alpha_{\mathrm{i}}\right)$ and their standard deviations (vertical bars) of the voxels from Fig. 8. The histograms below each plot show the spread of values of $\alpha$ across all sessions. 
is important. For example, it is possible that spatial preprocessing may affect intersession variance quite independent of underlying physical or physiological variability. The realignment procedure used (Ashburner et al., 1997) seeks to minimize the sum-ofsquares differences between successive volumes and a reference (here, the first vol ume in the time series). It is a facile point that each paradigm induces intensity changes in voxels (i.e., it "activates" them), and so volumes acquired during the "on" period of each paradigm will contain focal intensity differences from vol umes acquired during "rest." As a successful realignment between two volumes relies on the volumes used being similar rigid bodies, differing only in their alignment in space, the paradigm-induced intensity changes will affect the efficacy of alignment and may ultimately raise intersession variance. In addition, similar effects in voxels lying at a tissue boundary (voxels in the walls of the ventricles, for example) may result from simple repositioning of the subject between sessions, causing session-specific partial volume effects.

Examples like the above make it difficult to conclude if the variability which we observe is attributable to differences in: (i) the scanning environment (e.g., position of subject within headcoil) or (ii) preprocessing (misalignment). We are more confident that we were able to minimize session-by-condition interactions that could be attributable to performance differences. We treated each successive scanning session as though it was the first time that our subject had been scanned, to examine the potential influence of session context on a single-session experiment (acknowledging that we cannot overcome the Groundhog Day effect). It could be argued that systematic differences in our subject's performance across sessions may have resulted in the session-bycondition interactions which we observed, as the repeated execution of any active task or protocol of sensory stimulation may result in habituation or learning effects (e.g., Karni et al., 1995). We chose our activation tasks to minimize this possibility. Our subject was pretrained on the motor task, and our task frequency was chosen to lie within a range previously demonstrated by Blinkenberg and colleagues (1996) to have a low error rate (between 1 and $2 \mathrm{~Hz}$ ). Similarly, we chose a stable rate of number generation for our cognitive task (informed by the results of J ahanshahi et al., submitted for publication). Although we did not record subject performance on this task (primarily because of the motion it would produce), performance at random number generation remains stable over a number of repetitions (Evans et al., 1980). Furthermore, a preliminary MANCOVA analysis of our motor data set examining the effects of session revealed no systematic expression of the experimental variance over subse- quent sessions (data not shown). Although we accept that learning effects may exhibit complex temporal dynamics, the structure of our parameter estimates over sessions suggests random variation (Fig. 8) around a "true" mean parameter estimate. However, without independent measures of task performance, we cannot entirely rule out between-session habituation or learning-related changes in activation.

\section{Stability of fMRI Results across Sessions- Consequences for Longitudinal Studies}

As noted previously, fMRI is ideally suited to the examination of learning or recovery-of-function studies. These studies are typically predicated on the assumption that the experimental effects will be large enough to ensure their detection compared to nonspecific between-session effects. Because of the considerable time that must typically be devoted to such studies, it would be useful to have some idea of the relative magnitudes of each effect before beginning. Although in the present study we have examined the similarity of the results between sessions while accepting a certain degree of difference in the practical implementation of each session, our results do not really address the issue of signal to noise in longitudinal fMRI studies. As any difference between sessions is a session-bycondition interaction, any study which purports to focus on session-by-condition interactions produced by the experimental manipulation must ensure that nonspecific session-by-condition interactions can be efficiently controlled (for a discussion of these issues, see Petersson et al., 1999). We examined three tasks that were designed to show limited session-by-condition interactions in subject performance. As such, our results cannot be used to address the validity of Iongitudinal fMRI studies. We note, however, that the stability of our results suggests that longitudinal studies that produce unambiguous results should be feasible.

\section{Use of Thresholded Statistical Maps to Analyze Session Generality}

Typically, the results of neuroimaging experiments are displayed using binarized statistical maps. In this fashion, voxels that pass a predetermined statistical threshold are classified as active and other voxels as inactive. Although the utility and clarity of the results motivate this approach, much of the richness of functional neuroimaging data sets is removed. Attempts to examine the test-retest reliability of fMRI using measures such as "voxel counting" on thresholded maps therefore suffer from two problems: an essentially arbitrarily defined statistical threshold and the loss of complexity which accompanies any method that has to classify voxels as either active or inactive. We sought, instead, to characterize our data sets in terms of the between-session variance of the activation effects and 
not merely examine when voxels passed an arbitrarily set threshold on successive sessions. The differences between the two approaches are apparent when one compares the results of our single-session analyses (Figs. 2, 3, and 4) with our later multisession analyses (Figs. 5, 6, and 7). Generally a failure to detect activation may say more about the sensitivity of the experiment than the presence of the effect itself (Poline et al ., 1996). Certain areas may therefore appear more variable than they truly are.

\section{Effects of Sample Size on the Analysis of Generality}

Our results demonstrate the need for a large sample size when examining how well a single fMRI session exemplifies a subject's responses. The plots of parameter estimates by session in Fig. 9 show that voxels in which we found significant session-by-condition interactions over all of our sessions appear surprisingly stable when examined over a small number of sessions (for example, sessions 27-29 in Fig. 9C are almost identical). This effect has been termed "the law of small numbers" (Tversky et al., 1971) — the tendency to ascribe a lack of variability to small sample groups. Our use of a large number of sessions allowed us to characterize variability that may have been missed by previous studies employing five repeated sessions at most on the same subject. However, the opposite argument may be level ed at our results: if the sample size is large enough, then a statistically significant difference will always be found-this is merely an example of the fallacy of classical hypothesis testing. We accept this criticism, but believe that an analysis of 30 sessions is an appropriate sample size for the purposes of this study. The existence of significantly variable voxels necessitates the use of a random-effects model to allow the experiments to truly generalize their results to the subject.

\section{Levels of Inference Arising from Fixed- and Random-Effects Models}

Worlsey and colleagues (1992) first suggested the use of a "summary statistic" approach to the analysis of functional neuroimaging data. However, the implementation we used in the current study is that of Holmes and Friston (1998), who suggested randomeffects analyses for balanced designs in neuroimaging employing a general linear framework to allow for the between-subject variance component in multisubject designs. As discussed previously, the random-effects analysis confers generality, but with a concomitant loss of sensitivity due to the inevitable low degrees of freedom. We assumed that the $\epsilon_{\mathrm{i}}$ were Normally distributed and incorporated this assumption into our random-effects level model. However, by examining Fig. 9 it is clear that the $\epsilon_{\mathrm{i}}$ do not necessarily conform to this distribution. If we examine the case of the voxel in Fig. 9A, it is clear that this voxel has a skewed-right distribution. Indeed, if one asks a simpler question of the voxel in Fig. 9A (how often is $\alpha>0$ ) and employ a simple sign test, the probability of getting $31 / 33$ positive $\alpha$ 's is $<7 \times 10^{-8}$. Yet this voxel does not pass the random-effects analysis used here. Although we accept that this is only one voxel, it casts doubts on assumptions of N ormality for the $\epsilon_{1}$, and it is clear that further investigation is needed into the distribution of between-session variance. The development of randomeffects models that do not require prior assumptions of the distribution of residuals may be needed to address this issue.

The use of random-effects models in the analysis of $\mathrm{fMRI}$ data is a recent addition to the canon of neuroimaging analysis methods, and it is wise to note a previous adoption of this measure. In the analysis of behavioral data from human subjects, Clark's (1973) initial proposal that the model should be used more frequently highlighted an obvious problem: treating a sample from a population as random and selecting a sample randomly from a population are clearly not the same. Although we could argue that by using a random-effects analysis in our study, we can generalize our results to our subject as a putative population, we have performed a very limited sampling of our subject's responses. Each scanning session was performed over a 2-month period only, and session times were selected in a biased manner: near midday and near 6 PM in the evening. However, it is not el egant to have to state that "our results generalize to the population of possible sessions sampled from our subject over a period of 2 months, using the resources available in our laboratory." In practice, these caveats are usually accepted. Indeed, the use of random-effects models to ensure the correct level of inference in multisubject fMRI analyses rarely addresses the other sources of systematic variation in the population that the investigators are generalizing to (usually male, Caucasian right-handers who respond to advertisements and financial reward). However, adopting a random-effects model does afford some protection against inappropriate generalization of results, as noted by Abelson (1995).

Although we have shown that with an appropriate statistical model and a large sample of sessions we can obtain robust results, a number of issues remain unanswered. In particular, we would hesitate before generalizing our own results to other centers, subjects, or activation paradigms, as between-session variance may vary greatly depending on the context under which it is studied.

\section{CONCLUSIONS}

In this paper we described the results of an experiment designed to examine intersession variance in $\mathrm{fMRI}$ during the performance of simple visual, motor, 
and cognitive tasks by a single subject. First, analyzing our data session by session, we suggested that binarized statistical maps, though convenient, are not a useful tool for the evaluation of intersession variability. We then described an analytical framework that allowed us to identify significantly variable voxels by session across our multisession data sets. Each multisession data set, by paradigm, showed evidence of significant session-by-condition interactions. This result demonstrates that session context effects have a significant effect on fMRI data and illustrates that a single session should be considered merely as a single sample of a subject's responses to the experimental intervention employed. As we sampled a large number of sessions across all paradigms, we then compared the differences between analyzing these data using either fixed- or random-effects linear models, the latter being a recent addition to neuroimaging analysis. Although we comment on the usefulness of random effects analyses, which allow inference about experimental effects to be extended to the population which the sessions were sampled from, we draw attention to the intersession distribution of voxel response amplitudes. Our assumption of Normally distributed intersession residuals was not supported by close examination of some of our data, and so we accept that future work is required before random-effects models can be used to their full potential. Finally, we acknowledge that identifying the source and magnitude of the different sources of intersession variance in fMRI is crucial. The ability to differentiate between variability caused by the neurovascular signals that fMRI measures, and variability introduced by the means of measurement and analysis of these signals, is essential for the future of $\mathrm{fMRI}$ as a noninvasive imaging modality.

\section{ACKNOWLEDGMENTS}

We thank J esper Andersson and J ohn Ashburner for invaluable help and advice and our reviewers for their constructive and generous comments. The Wellcome Trust supported this work.

\section{REFERENCES}

Abelson, R. P. 1995. Statistics as Principled Argument. Elbaum, Hillsdale, NJ .

Ashburner, J ., and Friston, K. J . 1999. Nonlinear spatial normalisation using basis functions. Hum. Brain Mapp. 7:254-266.

Ashburner, J ., Neelin, P., Collins, D. L., Evans, A. C., and Friston, K. J . 1997. Incorporating prior knowledge into image registration. Neurol mage 6:344-352.

Belliveau, J. W., Kennedy, D. N., McKinstry, R. C., Buchbinder, B. R., Weisskoff, R. M., Cohen, M. S., Vevea, J . M., Brady, T. J ., and Rosen, B. R. 1991. Functional mapping of the human visualcortex by magnetic-resonance-imaging. Science 254:716-719.

Blinkenberg, M., Bonde, C., Holm, S., Svarer, C., Andersen, J., Paulson, O. B., and Law, I. 1996. Rate dependence of regional cerebral activation during performance of a repetitive motor task: A PET study. J . Cereb. Blood Flow Metab. 16:794-803.
Casey, B. J ., Cohen, J . D., O'Craven, K., Davidson, R. J ., Irwin, W., Nelson, C. A., Noll, D. C., Hu, X., Lowe, M. J., Rosen, B. R., Truwitt, C. L., and Turski, P. A. 1998. Reproducibility of fMRI results across four institutions using a spatial working memory task. Neurol mage 8:249-261.

Clark, H. H. 1973. The language-as-fixed-effect fallacy. J . Verb. Learn. Verb. Behav. 12:335-359.

Cohen, M. S., and DuBois, R. M. 1999. Stability, repeatability, and the expression of signal magnitude in functional magnetic resonance imaging. J. Magn. Reson. Imag. 10:33- 40.

Draper, N. R., and Smith, H. 1981. Applied Regression Analysis, pp. 97-98. Wiley, New York.

Evans, A. C., Collins, D. L., Mills, S. R., Brown, E. D., Kelly, R. L., and Peters, T. M. 1993. 3D statistical neuroanatomical models from $305 \mathrm{MRI}$ volumes. In Proceedings, IEEE Nuclear Science Symposium and Medical Imaging Conference, pp. 1813-1817. IEEE Inc., Piscataway, NJ .

Evans, F. J ., and Graham, C. 1980. Subjective random number generation and attention deployment during acquisition and overlearning of a motor skill. Bull. Psychonom. Soc. 15:391-394.

Fox, P. T., and Raichle, M. E. 1985. Stimulus rate determines re gional blood flow in striate cortex demonstrated by positron emission tomography. Ann. Neurol. 17:303-305.

Frison, L., and Pocock, S. J. 1992. Repeated measures in clinical trials: An analysis using mean summary statistics and its implications for design. Stat. Med. 11:1685-1704.

Friston, K. J ., Ashburner, J ., Frith, C. D., Poline, J .-B., Heather, J. D., and Frackowiak, R. S. J. 1995. Spatial registration and normalisation of images. Hum. Brain Mapp. 2:165-189.

Genovese, C. R., Noll, D. C., and Eddy, W. F. 1997. Estimating test-retest reliability in functional $M R$ imaging. I. Statistical methodology. Magn. Res. Med. 38:497-507.

Grady, C. L., Van Meter, J . W., Maisog, J . M., Pietrini, P., Krasuski, J ., and Rauschecker, J. P. 1997. Attention-related modulation of activity in primary and secondary auditory cortex. NeuroReport 28:2511-2516.

Henson, R. N. A., Shallice, T., and Dolan, R. J . 1999a. Right prefrontal cortex and episodic memory retrieval: A functional MRI test of the monitoring hypothesis. Brain 122:1367-1381.

Henson, R. N. A., Rugg, M. D., Shallice, T., J osephs, O., and Dolan, R. J . 1999b. Recollection and familiarity in recognition memory: An event-related functional magnetic resonance imaging study. J . Neurosci. 19:3962-3972.

Holmes, A. P., J osephs, O., Büchel, C., and Friston, K. J. 1997. Statistical modeling of low frequency confounds in fMRI. NeuroI mage 5:S480.

Holmes, A. P., and Friston, K. J . 1998. Generalisability, random effects and population inference. Neurol mage 7:S754.

Howseman, A. M., McGonigle, D. J ., Grootoonk, S., Ramdeen, J ., Athwal, B. S., and Turner, R. 1998. Assessment of the variability in $\mathrm{fMRI}$ data sets due to subject positioning and calibration of the MRI scanner. Neurol mage 7:S599.

Karni, A., Meyer, G., J ezzard, P., Adams, M. M., Turner, R., and Ungerleider, L. G. 1995. Functional MRI evidence for adult motor cortex plasticity during motor skill learning. Nature 377:155-158.

Kwong, K. K., Belliveau, J. W., Chesler, D. A., Goldberg, I. E., Weisskoff, R. M., Poncelet, B. P., Kennedy, D. N., Hoppel, B. E., Cohen, M. S., Turner, R., Cheng, H. M., Brady, T. J ., and Rosen, B. R. 1992. Dynamic magnetic resonance imaging of human brain activity during primary sensory stimulation. Proc. Natl. Acad. Sci. USA 89:5675-5679.

Le, T. H., and Hu, X. 1997. Methods for assessing accuracy and reliability in functional MRI. NMR Biomed. 10:160-164. 
Marinković, R., Cvejin, B., Marković, L., and Budimlija, Z. 1995. [Morphologic characteristics of the vascular network in the striate area in humans]. Med. Pregl. 48:7-9.

Noll, D. C., Genovese, C. R., Nystrom, L. E., Vazquez, A. L., Forman, S. D., Eddy, W. F., and Cohen, J . D. 1997. Estimating test-retest reliability in functional MR imaging. II. Application to motor and cognitive activation studies. Magn. Res. Med. 38:508-517.

Ogawa, S., Menon, R. S., Tank, D. W., Kim, S. G., Merkle, H., Ellerman, J. M., and Urgubil, K. 1992. Intrinsic signal changes accompanying sensory stimulation: Functional brain mapping with magnetic resonance imaging. Proc. Natl. Acad. Sci. USA 89:5951-5955.

Ogawa, S., Menon, R. S., Kim, S.-G., and Ugurbil, K. 1998. On the characteristics of functional magnetic resonance imaging of the brain. Annu. Rev. Biophys. Biomol. Struct. 27:447- 474.

Ojemmaan, J. G., Buckner, R. L., Akbudak, E., Snyder, A. Z., Ollinger, J. M., McKinstry, R. C., Rosen, B. R., Petersen, S. E., Raichle, M. E., and Conturo, T. E. 1998. Functional MRI studies of word-stem completion: Reliability across laboratories and comparison to blood flow imaging with PET. Hum. Brain Mapp. 6:203215.

Petersson, K. M., Elfgren, C., and Ingvar, M. 1999. Learning-related effects and functional neuroimaging. Hum. Brain Mapp. 7:234243.

Poline, J . B., Vandenberghe, R., Holmes, A. P., Friston, K. J ., and Frackowiak, R. S. J . 1996. Reproducibility of PET activation studies: Lessons from a multi-center European experiment. Neurol mage 4:34-54.

Rao, S. M., Binder, J . R., Bandettini, P. A., Hammeke, T. A., Yetkin, F. Z., J esmanowicz, A., Lisk, L. M., Morris, G. L., Mueller, W. M.,
Estkowski, L. D., Wong, E. C., Haughton, V. M., and Hyde, J. S. 1993. Functional magnetic resonance imaging of complex human movements. Neurology 43:2311-2318.

Rombouts, S. A. R. B., Barkhof, F., Hoogenraad, F. G. C., Sprenger, M., and Scheltens, P. 1998. Within-subject reproducibility of visual activation patterns with functional magnetic resonance imaging using multislice echo planar imaging. Magn. Reson. I mag. 16:105113.

Searle, S. R., Casella, G., and McCulloch, C. E. 1992. Variance Components. Wiley, New York.

Talairach, P., and Tournoux, J . 1988. A Stereotactic Coplanar Atlas of the Human Brain. Thieme, Stuttgart.

Tegeler, C., Strother, S. C., Anderson, J. R., and Kim, S. G. 1999. Reproducibility of BOLD-based functional MRI obtained at 4T. Hum. Brain Mapp. 7:267-283.

Tversky, A., and Kahneman, D. 1971. Belief in the 'law of small numbers.' Psychol. Bull. 75:105-110.

Woodruff, P. W. R., Benson, R. R., Bandettini, P. A., Kwong, K. K., Howard, R. J ., Talavage, T., Belliveau, J ., and Rosen, B. R. 1996. Modulation of auditory and visual cortex by selective attention is modality-dependent. NeuroReport 7:1909-1913.

Worsley, K. J ., Evans, A. C., Marrett, S., and Neelin, P. 1992. A three-dimensional statistical analysis for CBF activation studies in human brain. J . Cereb. Blood Flow Metab. 12:900-918.

Worsley, K. J ., and Friston, K. J . 1995. Analysis of fMRI timeseries revisited-Again. Neurol mage 2:173-181.

Yetkin, F. Z., McAuliffe, T. L., Cox, R., and Haughton, V. M. 1996. Test-retest precision of functional MR in sensory and motor task activation. AJ NR 17:195-198. 